\title{
Mathematical modeling of the immune-chemotherapeutic treatment of breast cancer under some control parameters
}

\author{
Ali Yousef ${ }^{1 *}$ (D), Fatma Bozkurt ${ }^{1,2}$ and Thabet Abdeljawad ${ }^{3,4}$
}

\section{"Correspondence:}

a.yousef@kcst.edu.kw

'Department of Mathematics,

Kuwait College of Science and

Technology, 27235 Kuwait City, Kuwait

Full list of author information is available at the end of the article

\section{Springer}

\begin{abstract}
We consider a system of fractional-order differential equations to analyze breast cancer growth in the immune-chemotherapeutic treatment process under some control parameters: ketogenic diet, immune booster, and anti-cancer drugs. The established model assumes the growth of the tumor density under chemotherapy treatment and the immune response during the interaction between the normal cells and tumor cells. For the local stability of the critical points (tumor-free critical point, dead critical point, and co-existing critical point), we used the Routh-Hurwitz criteria to show the necessary effect of the immune booster; moreover, we addressed the ketogenic rate in the treatment process. Our theoretical and numerical studies pointed out that on early detection of the tumor density (with weak Allee effect) the treatment should be supported by ketogenic nutrition. Several examples are shown to present our theoretical findings.
\end{abstract}

Keywords: Fractional-order differential equations; Stability analysis; Allee effect; Breast cancer; Immune-chemotherapeutic treatment

\section{Introduction}

According to the National Cancer Registry, cancer kills more people than tuberculosis, AIDS, and malaria combined. Statistics show that cancer mortality is estimated at 13 million deaths by 2030 [1-3]. Breast cancer is the most widely recognized and obstructive disease in females around the world. Breast cancer is a disease that affects the breast's cells and causes an uncontrolled division of abnormal cells that can be malignant in the breast tissues [4]. Some of the breast cancer risk factors are hormonal imbalance, genetics, and environmental; however, there is still little information on this disease as regards the leading cause of a malignant form $[5,6]$. Various mathematical and analytical approaches have been developed to understand the interaction between the tumor cells and the immune response during the treatment process that was mainly established as integer-order differential equations (IDEs) [7-9].

However, it is seen that many problems in biology, as well as in other fields such as engineering, finance, economics, and neural networks, can be formulated successfully by fractional-order differential equations (FDEs); see, for instance, Refs. [10-15]. The nonlo-

0 The Author(s) 2020. This article is licensed under a Creative Commons Attribution 4.0 International License, which permits use sharing, adaptation, distribution and reproduction in any medium or format, as long as you give appropriate credit to the original author(s) and the source, provide a link to the Creative Commons licence, and indicate if changes were made. The images or other third party material in this article are included in the article's Creative Commons licence, unless indicated otherwise in a credit line to the material. If material is not included in the article's Creative Commons licence and your intended use is not permitted by statutory regulation or exceeds the permitted use, you will need to obtain permission directly from the copyright holder. To view a copy of this licence, visit http://creativecommons.org/licenses/by/4.0/ 
cal property of models of FDEs is not only depending on the current state but also provides an adequate description for the historical ones. The transformation of models governed by IDES into models of FDE needs to be precise due to the peculiarity of the order of differentiation $\alpha$, where a small change in $\alpha$ may cause a significant change in the result. FDEs can model certain phenomena that cannot be modeled by IDEs. Thus, FDEs mainly use biological models since they are relevant to memory and the hereditary feature [16-20].

\section{Mathematical model}

Over the years, modeling breast cancer has become a valuable tool to understand tumor growth's dynamical behavior in the treatment process. D'Onofrio et al. explored the role of mathematical modeling in combination therapy for tumors [21]. Studies of Kermack and McKendrick [22, 23] and some other investigations in [24-30] have shown that modeling mathematically the biological phenomena is a useful tool to solve epidemical problems.

However, these studies did not include a nutritional diet (ketogenic diet) in their mathematical model. Oke et al. improved the model of Mudufza [28] in [3]. They incorporated the control parameters such as ketogenic diet, immune booster, and anti-cancer drug to emphasize the point that there is an interaction between the cells due to the mutation in the tumor cell's DNA.

In this paper, we establish a system of FDEs in the form

$$
\left\{\begin{aligned}
D^{\alpha} N(t)= & N(t) r_{1}\left(K_{1}-\mu_{1} N(t)-\beta_{1} T(t)\right)-(1-k) \phi_{1} N(t) E(t), \\
D^{\alpha} T(t)= & T(t) r_{2}\left(K_{2} d-\mu_{2} T(t)-\beta_{2} I(t)\right)-\delta T(t) \\
& +(1-k) \phi_{1} N(t) E(t) T(t), \\
D^{\alpha} I(t)= & \rho \gamma+I(t)\left(K_{3}-\mu_{3}-\beta_{3} T(t)\right)-(1-k) \phi_{2} I(t) E(t), \\
D^{\alpha} E(t)= & (1-k) \varepsilon-\beta_{4} E(t),
\end{aligned}\right.
$$

where the parameters denote positive real numbers.

The first equation in system (2.1) represents the normal cell population $N(t) . r_{1}$ is the growth rate, while $K_{1}$ is the carrying capacity of the population. $\mu_{1}$ represents the logistic rate and $\beta_{1}$ denotes the inhibition rate of $N(t) . \phi_{1}$ is the tumor formation rate resulting from DNA mutation caused by the presence of excess estrogen, while $(1-k)$ represents the effectiveness of anti-cancer drugs.

In the second equation of (2.1), we show the luminal type (having estrogen receptors, ESR1 + ve) tumor cells that are denoted by $T(t) . K_{2}$ is the carrying capacity of the population, and it depends on the rate of $d$, which is the ketogenic diet. $r_{2}$ is the growth rate of the $T(t)$ population. Any mutation in DNA that is caused by excess estrogen repopulate the tumor cells by $\phi_{1} N(t) E(t) . \mu_{2}$ represents the logistic rate of the tumor cell population. $\beta_{2}$ is the rate of the effectiveness of the immune system to the tumor cells, $\delta$ is the result of the tumor starvation nutrients during the ketogenic diet.

The third equation in (2.1) shows the class of immune response as $I(t)$, where $\rho$ denotes the source rate of immune response fully infused in the body daily. The immune booster $\gamma$ assists the immune response whenever tumor cells overpower immune cells to activate the immune response and fight against the tumor cells. $\mu_{3}$ represents the logistic rate of the immune cell population, while $\phi_{2}$ is the immune suppression rate. $K_{3}$ is the carrying capacity and $\beta_{3}$ is the rate of interaction between $T(t)$ and $I(t)$. 
Table 1 The description of parameters

\begin{tabular}{|c|c|c|c|}
\hline Parameter & Symbol & Value & Unit \\
\hline The growth rate of $\mathrm{NC}$ & $r_{1}$ & 0.3 & day $^{-1}$ \\
\hline The growth rate of $\mathrm{TC}$ & $r_{2}$ & 0.4 & day $^{-1}$ \\
\hline Logistic rate of $\mathrm{NC}$ & $\mu_{1}$ & {$[0.05-0.2]$} & day $^{-1}$ \\
\hline Logistic rate of $\mathrm{TC}$ & $\mu_{2}$ & {$[0.5-0.95]$} & day $^{-1}$ \\
\hline Logistic rate of IC & $\mu_{3}$ & {$[0.05-0.2]$} & day $^{-1}$ \\
\hline Carrying capacity of NC & $K_{1}$ & 1.232 & day $^{-1}$ \\
\hline Carrying capacity of TC & $K_{2}$ & 1.75 & day $^{-1}$ \\
\hline Carrying capacity of IC & $K_{3}$ & {$[0.11,1.17]$} & day $^{-1}$ \\
\hline Constant rate of the ketogenic diet & $d$ & 0.5 & day $^{-1}$ \\
\hline Rate of inhibition of NC & $\beta_{1}$ & $6 \times 10^{-8}$ & day $^{-1}$ \\
\hline TC death rate due to immune response & $\beta_{2}$ & $3 \times 10^{-6}$ & day $^{-1}$ \\
\hline Interaction coefficient rate with immune response & $\beta_{3}$ & $1 \times 10^{-7}$ & day $^{-1}$ \\
\hline The natural death rate of estrogen & $\beta_{4}$ & 0.97 & day $^{-1}$ \\
\hline Tumor formation rate as a result of DNA damage by excess estrogen & $\phi_{1}$ & 0.2 & day $^{-1}$ \\
\hline Immune suppression rate due to excess estrogen & $\phi_{2}$ & 0.002 & day $^{-1}$ \\
\hline Efficacy of the anti-cancer drug & $k$ & $0-1$ & day $^{-1}$ \\
\hline Source rate of IC & $\rho$ & $1.3 \times 10^{2}$ & day $^{-1}$ \\
\hline Supplement of immune booster & $\gamma$ & 0.01 & day $^{-1}$ \\
\hline The death rate of TC due to the ketogenic diet & $\delta$ & 2 & day $^{-1}$ \\
\hline Source rate of estrogen & $\varepsilon$ & {$[0.6-3]$} & day $^{-1}$ \\
\hline
\end{tabular}

Finally, the last equation of system (2.1) is the estrogen compartment, which is denoted by $E(t)$. It should be known that an increase in estrogen level can lead to the growth of the tumor cells. The process of constantly replenishing excess estrogen is denoted by $\varepsilon$. We assume that the majority of cancer cells are estrogen-receptor positive and only a small proportion of epithelial cells are estrogen-receptor positive, which can only be blocked by the anti-cancer drug $(1-k)$ tamoxifen. $\beta_{4}$ is the rate at which estrogen is being washed out from the body $[3,22]$. According to the information on the parameters, all parametric values are stated in Table 1.

Definition 2.1 ([31]) Given a function $\varphi(x)$, the fractional integral with order $\alpha>0$ is given by Abdel's formula by

$$
I_{\alpha} \varphi(x)=\frac{1}{\Gamma(\alpha)} \int_{0}^{x} \varphi(t)(x-t)^{\alpha-1} f(t) d t, \quad x>0 .
$$

Definition 2.2 ([31]) Let $\varphi: R^{+} \rightarrow R$ be a continuous function. The Caputo fractional derivative of order $\alpha \in(n-1, n)$, where $n$ is a positive integer is defined as

$$
D_{0}^{\alpha} \varphi(x)=\frac{1}{\Gamma(n-\alpha)} \int_{0}^{x} \frac{\varphi^{(n)}(t)}{(x-t)^{\alpha+1-n}} d t .
$$

When $\alpha=n$, the derivatives are defined to be the usual $n$th order derivatives.

Definition 2.3 ([32]) The Mittag-Leffler function of one variable is

$$
E_{\alpha}(\lambda, z)=E_{\alpha}\left(\lambda z^{\alpha}\right)=\sum_{k=0}^{\infty} \frac{\lambda^{k} z^{\alpha k}}{\Gamma(1+\alpha k)} \quad(\lambda \neq 0, z \in \mathbb{C}: \operatorname{Re}(\alpha)>0) .
$$




\section{The equilibrium points}

Consider the system

$$
\left\{\begin{aligned}
D^{\alpha} N(t) & =f(N(t), T(t), I(t), E(t)) \\
& =N(t) r_{1}\left(K_{1}-\mu_{1} N(t)-\beta_{1} T(t)\right)-(1-k) \phi_{1} N(t) E(t) \\
D^{\alpha} T(t) & =g(N(t), T(t), I(t), E(t)) \\
& =T(t) r_{2}\left(K_{2} d-\mu_{2} T(t)-\beta_{2} I(t)\right)-\delta T(t)+(1-k) \phi_{1} N(t) E(t) T(t) \\
D^{\alpha} I(t) & =h(N(t), T(t), I(t), E(t)) \\
& =\rho \gamma+I(t)\left(K_{3}-\mu_{3}-\beta_{3} T(t)\right)-(1-k) \phi_{2} I(t) E(t) \\
D^{\alpha} E(t) & =j(N(t), T(t), I(t), E(t))=(1-k) \varepsilon-\beta_{4} E(t) .
\end{aligned}\right.
$$

To analyze the stability of the system (3.1), we perturb the equilibrium point by adding $\varepsilon_{i}(t)>0, i=1,2,3,4$, that is,

$$
\begin{aligned}
& N(t)-\bar{N}=\varepsilon_{1}(t), \quad T(t)-\bar{T}=\varepsilon_{2}(t), \\
& I(t)-\bar{I}=\varepsilon_{3}(t) \quad \text { and } \quad E(t)-\bar{E}=\varepsilon_{4}(t) .
\end{aligned}
$$

Thus, we have

$$
\begin{aligned}
D^{\alpha}\left(\varepsilon_{1}(t)\right) \simeq & f(\bar{N}, \bar{T}, \bar{I}, \bar{E})+\frac{\partial f(\bar{N}, \bar{T}, \bar{I}, \bar{E})}{\partial N} \varepsilon_{1}(t)+\frac{\partial f(\bar{N}, \bar{T}, \bar{I}, \bar{E})}{\partial T} \varepsilon_{2}(t) \\
& +\frac{\partial f(\bar{N}, \bar{T}, \bar{I}, \bar{E})}{\partial I} \varepsilon_{3}(t)+\frac{\partial f(\bar{N}, \bar{T}, \bar{I}, \bar{E})}{\partial j} \varepsilon_{4}(t), \\
D^{\alpha}\left(\varepsilon_{2}(t)\right) \simeq & g(\bar{N}, \bar{T}, \bar{I}, \bar{E})+\frac{\partial g(\bar{N}, \bar{T}, \bar{I}, \bar{E})}{\partial N} \varepsilon_{1}(t)+\frac{\partial g(\bar{N}, \bar{T}, \bar{I}, \bar{E})}{\partial T} \varepsilon_{2}(t) \\
& +\frac{\partial g(\bar{N}, \bar{T}, \bar{I}, \bar{E})}{\partial I} \varepsilon_{3}(t)+\frac{\partial g(\bar{N}, \bar{T}, \bar{I}, \bar{E})}{\partial j} \varepsilon_{4}(t), \\
D^{\alpha}\left(\varepsilon_{3}(t)\right) \simeq & h(\bar{N}, \bar{T}, \bar{I}, \bar{E})+\frac{\partial h(\bar{N}, \bar{T}, \bar{I}, \bar{E})}{\partial N} \varepsilon_{1}(t)+\frac{\partial h(\bar{N}, \bar{T}, \bar{I}, \bar{E})}{\partial T} \varepsilon_{2}(t) \\
& +\frac{\partial h(\bar{N}, \bar{T}, \bar{I}, \bar{E})}{\partial I} \varepsilon_{3}(t)+\frac{\partial h(\bar{N}, \bar{T}, \bar{I}, \bar{E})}{\partial j} \varepsilon_{4}(t)
\end{aligned}
$$

and

$$
\begin{aligned}
D^{\alpha}\left(\varepsilon_{4}(t)\right) \simeq & j(\bar{N}, \bar{T}, \bar{I}, \bar{E})+\frac{\partial j(\bar{N}, \bar{T}, \bar{I}, \bar{E})}{\partial N} \varepsilon_{1}(t)+\frac{\partial j(\bar{N}, \bar{T}, \bar{I}, \bar{E})}{\partial T} \varepsilon_{2}(t) \\
& +\frac{\partial j(\bar{N}, \bar{T}, \bar{I}, \bar{E})}{\partial I} \varepsilon_{3}(t)+\frac{\partial j(\bar{N}, \bar{T}, \bar{I}, \bar{E})}{\partial j} \varepsilon_{4}(t) .
\end{aligned}
$$

This perturbation around the equilibrium point is to linearize the system since we established a nonlinear fractional-order system based on the Lotka-Volterra logistic equation. Moreover, since we are working with the Caputo derivative, the constant function is here zero. 
We use the fact that $f(\bar{N}, \bar{T}, \bar{I}, \bar{E})=g(\bar{N}, \bar{T}, \bar{I}, \bar{E})=h(\bar{N}, \bar{T}, \bar{I}, \bar{E})=j(\bar{N}, \bar{T}, \bar{I}, \bar{E})=0$ and obtain, therefore, a linearized system about the equilibrium point such as

$$
D^{\alpha} Z=J Z
$$

where $Z=\left(\varepsilon_{1}(t), \varepsilon_{2}(t), \varepsilon_{3}(t), \varepsilon_{4}(t)\right)$. Moreover, $J$ is the Jacobian matrix at equilibrium:

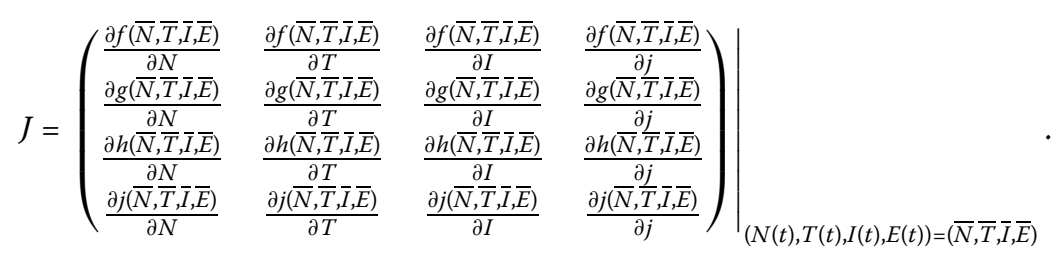

We have $B^{-1} J B=C$, where $C$ is the diagonal matrix of the eigenvalues $\lambda_{i}(i=1,2,3,4)$ and $B$, the eigenvectors of $J$. Therefore, we get

$$
\left\{\begin{array}{l}
D_{0}^{\alpha} \eta_{1}=\lambda_{1} \eta_{1} \\
D_{0}^{\alpha} \eta_{2}=\lambda_{2} \eta_{2}, \\
D_{0}^{\alpha} \eta_{3}=\lambda_{3} \eta_{3}, \\
D_{0}^{\alpha} \eta_{4}=\lambda_{4} \eta_{4}
\end{array} \quad \text { where } \eta=\left(\begin{array}{l}
\eta_{1} \\
\eta_{2} \\
\eta_{3} \\
\eta_{4}
\end{array}\right) \text { and } \eta=B^{-1} Z\right.
$$

whose solutions are given by the Mittag-Leffler functions,

$$
\begin{aligned}
& \eta_{1}(t)=\sum_{n=0}^{\infty} \frac{\left(\lambda_{1}\right)^{n} t^{n \alpha}}{\Gamma(n \alpha+1)} \eta_{1}(0)=E_{\alpha}\left(\lambda_{1} t^{\alpha}\right) \eta_{1}(0), \\
& \eta_{2}(t)=\sum_{n=0}^{\infty} \frac{\left(\lambda_{2}\right)^{n} t^{n \alpha}}{\Gamma(n \alpha+1)} \eta_{2}(0)=E_{\alpha}\left(\lambda_{2} t^{\alpha}\right) \eta_{2}(0), \\
& \eta_{3}(t)=\sum_{n=0}^{\infty} \frac{\left(\lambda_{3}\right)^{n} t^{n \alpha}}{\Gamma(n \alpha+1)} \eta_{3}(0)=E_{\alpha}\left(\lambda_{3} t^{\alpha}\right) \eta_{3}(0)
\end{aligned}
$$

and

$$
\eta_{4}(t)=\sum_{n=0}^{\infty} \frac{\left(\lambda_{4}\right)^{n} t^{n \alpha}}{\Gamma(n \alpha+1)} \eta_{4}(0)=E_{\alpha}\left(\lambda_{4} t^{\alpha}\right) \eta_{4}(0) .
$$

Matington studied in [33], a fractional differential system involving the Caputo derivative

$$
D_{0}^{\alpha} x(t)=A x(t)
$$

with initial values $x(0)=x_{0}=\left(x_{10}, x_{20}, \ldots, x_{n 0}\right)^{T}$, where $x=\left(x_{1}, x_{2}, \ldots, x_{n}\right)^{T}, \alpha \in(0,1)$ and $A \in \mathbb{R}^{n \times n}$. The stability of system (3.10) was defined by Matington as follows.

Definition 3.1 The autonomous system (3.10) is said to be

(i) stable if and only if for any $x_{0}$, there exists $\varepsilon>0$ such that $\|x\| \leq \varepsilon$ for $t \geq 0$, 
(ii) asymptotically stable if and only if $\lim _{t \rightarrow \infty}\|x\|=0$.

Theorem A ([34]) The autonomous system (3.10) is

(i) asymptotically stable if and only if if $\left|\arg \left(\lambda_{i}\right)\right|>\frac{\alpha \pi}{2}$, in this case, the components of the state decay towards 0 like $t^{-\alpha}$,

(ii) stable if and only if either it is asymptotically stable, or those critical values which satisfy $\left|\arg \left(\lambda_{i}\right)\right|=\frac{\alpha \pi}{2}$ have geometric multiplicity one.

(Here, $\arg \left(\lambda_{i}\right)$ denotes the arguments of the eigenvalues of the square matrix $A$.)

The proof of Theorem A was sketched by Matington in [33], while Zeng et al. proved the theorem in detail by using the Mittag-Leffler functions in [35]. Therefore, considering system (3.3) we can say that if $\left|\arg \left(\lambda_{i}\right)\right|>\frac{\alpha \pi}{2}(i=1,2,3,4)$, then $\eta_{i}(t)(i=1,2,3,4)$ are decreasing and therefore also $\varepsilon_{i}(t)(i=1,2,3,4)$ are decreasing. Thus, let the solution $\left(\varepsilon_{1}(t), \varepsilon_{2}(t), \varepsilon_{3}(t), \varepsilon_{4}(t)\right)$ of (3.3) exist. If the solution of (3.3) is increasing, then $(\bar{N}, \bar{T}, \bar{I}, \bar{E})$ is unstable and if $\left(\varepsilon_{1}(t), \varepsilon_{2}(t), \varepsilon_{3}(t), \varepsilon_{4}(t)\right)$ is decreasing, then $(\bar{N}, \bar{T}, \bar{I}, \bar{E})$ is locally asymptotically stable.

There are four critical points; two of them are the dead critical points, one is a tumorfree critical point, and the other one is the co-existing critical point. These are given as follows:

Tumor-free critical point: $\Lambda_{1}=\left(\bar{N}_{1}, 0, \bar{I}_{1}, \bar{E}_{1}\right)=\left(\frac{r_{1} K_{1} \beta_{4}-(1-k)^{2} \phi_{1} \varepsilon}{\mu_{1} r_{1} \beta_{4}}, 0, \frac{\rho \gamma \beta_{4}}{(1-k)^{2} \phi_{2} \varepsilon-\beta_{4}\left(K_{3}-\delta\right)}, \frac{(1-k) \varepsilon}{\beta_{4}}\right)$, where $\phi_{1}<\frac{K_{1} r_{1} \beta_{4}}{\varepsilon(1-k)^{2}}$ and $\phi_{2}>\frac{\left(K_{3}-\delta\right) \beta_{4}}{\varepsilon(1-k)^{2}}$ for $K_{3}>\delta$.

Dead critical point 1: $\Lambda_{2}=\left(0,0, \bar{I}_{2}, \bar{E}_{2}\right)=\left(0,0, \frac{\rho \gamma \beta_{4}}{(1-k)^{2} \phi_{2} \varepsilon-\beta_{4}\left(K_{3}-\delta\right)}, \frac{(1-k) \varepsilon}{\beta_{4}}\right)$, where $\phi_{2}>\frac{\left(K_{3}-\delta\right) \beta_{4}}{\varepsilon(1-k)^{2}}$ for $K_{3}>\delta$.

Dead critical point $2: \Lambda_{3}=\left(0, \bar{T}_{3}, \bar{I}_{3}, \bar{E}_{3}\right)=\left(0, \bar{T}_{3}, \bar{I}_{3}, \frac{(1-k) \varepsilon}{\beta_{4}}\right)$, where $\phi_{2}>\frac{\left(K_{3}-\delta\right) \beta_{4}}{\varepsilon(1-k)^{2}}$ for $K_{3}>\delta$.

Co-existing critical point: $\Lambda_{4}=\left(\bar{N}_{4}, \bar{T}_{4}, \bar{I}_{4}, \bar{E}_{4}\right)$, where all classes are positive, where $\phi_{1}<$ $\frac{K_{1} r_{1} \beta_{4}}{\varepsilon(1-k)^{2}}$ and $\phi_{2}>\frac{\left(K_{3}-\delta\right) \beta_{4}}{\varepsilon(1-k)^{2}}$ for $K_{3}>\delta$.

\section{Stability, existence, and uniqueness}

This section analyzes the local stability of (3.1) around each obtained critical point. The Jacobian matrix of the tumor-free critical point $\Lambda_{1}=\left(\bar{N}_{1}, 0, \bar{I}_{1}, \bar{E}_{1}\right)$ is given as

$$
J\left(\Lambda_{1}\right)=\left(\begin{array}{cccc}
a_{11} & a_{12} & 0 & a_{14} \\
0 & a_{22} & 0 & 0 \\
0 & a_{32} & a_{33} & a_{34} \\
0 & 0 & 0 & a_{44}
\end{array}\right)
$$

where

$$
\begin{aligned}
& a_{11}=r_{1} K_{1}-2 \mu_{1} \bar{N}_{1}-\frac{(1-k)^{2} \phi_{1} \varepsilon}{\beta_{4}}, \quad a_{12}=-\beta_{1} r_{1} \bar{N}_{1}, \quad a_{14}=-(1-k) \phi_{1} \bar{N}_{1}, \\
& a_{22}=r_{2} K_{2} d-\delta-\beta_{2} r_{2} \bar{I}_{1}+\frac{(1-k)^{2} \phi_{1} \varepsilon}{\beta_{4}} \bar{N}_{1}, \quad a_{32}=-\beta_{3} \bar{I}_{1}, \\
& a_{33}=-\frac{\left((1-k)^{2} \phi_{2} \varepsilon-\beta_{4}\left(K_{3}-\mu_{3}\right)\right)}{\beta_{4}}, \quad a_{34}=-(1-k) \phi_{2} \bar{I}_{1}, \quad a_{44}=-\beta_{4} .
\end{aligned}
$$

On the other hand, the characteristic equation of $\Lambda_{1}$ is given by

$$
\left(a_{11}-\lambda\right)\left(a_{22}-\lambda\right)\left(a_{33}-\lambda\right)\left(a_{44}-\lambda\right)=0 .
$$


Theorem 4.1 Let $\Lambda_{1}$ be the tumor-free critical point of system (3.1) and assume that $\phi_{1}<$ $\frac{K_{1} r_{1} \beta_{4}}{\varepsilon(1-k)^{2}}$ and $\phi_{2}>\frac{\left(K_{3}-\delta\right) \beta_{4}}{\varepsilon(1-k)^{2}}$ hold. Then $\Lambda_{1}$ is stable local asymptotic if and only if

$$
\bar{N}_{1}<\frac{\left(\delta-r_{2} K_{2} d\right) \beta_{4}}{(1-k)^{2} \phi_{1} \varepsilon}
$$

where $r_{2}<\frac{\delta}{K_{2} d}$.

Proof From (4.2), it follows that

(i) $\lambda_{1}=r_{1} K_{1}-2 \mu_{1} \bar{N}_{1}-\frac{(1-k)^{2} \phi_{1} \varepsilon}{\beta_{4}}<0$ since $\phi_{1}<\frac{K_{1} r_{1} \beta_{4}}{\varepsilon(1-k)^{2}}$,

(ii) $\lambda_{2}=r_{2} K_{2} d-\delta-\beta_{2} r_{2} \bar{I}_{1}+\frac{(1-k)^{2} \phi_{1} \varepsilon}{\beta_{4}} \bar{N}_{1}<0 \Rightarrow \bar{N}_{1}<\frac{\left(\delta-r_{2} K_{2} d\right) \beta_{4}}{(1-k)^{2} \phi_{1} \varepsilon}$, where $r_{2}<\frac{\delta}{K_{2} d}$,

(iii) $\lambda_{3}=-\frac{\left((1-k)^{2} \phi_{2} \varepsilon-\beta_{4}\left(K_{3}-\mu_{3}\right)\right)}{\beta_{4}}<0$, since $\phi_{2}>\frac{\left(K_{3}-\delta\right) \beta_{4}}{\varepsilon(1-k)^{2}}$,

(iv) $\lambda_{4}=-\beta_{4}<0$.

Remark 4.1 In Theorem 4.1. it is seen that any increase in the ketogenic rate affects the growth of the tumor population. It is assumed here that the invasion of the tumor cells into the normal cells is small. Thus, the tumor cell population can be eliminated from the breast tissues, since $\Lambda_{1}$ depends on the immune response and the estrogen level.

The Jacobian matrix of the dead critical point $\Lambda_{2}=\left(0,0, \frac{\rho \gamma \beta_{4}}{(1-k)^{2} \phi_{2} \varepsilon-\beta_{4}\left(K_{3}-\delta\right)}, \frac{(1-k) \varepsilon}{\beta_{4}}\right)$ is given as

$$
J\left(\Lambda_{2}\right)=\left(\begin{array}{cccc}
a_{11} & 0 & 0 & 0 \\
0 & a_{22} & 0 & 0 \\
0 & a_{32} & a_{33} & a_{34} \\
0 & 0 & 0 & a_{44}
\end{array}\right)
$$

where

$$
\begin{aligned}
& a_{11}=r_{1} K_{1}-\frac{(1-k)^{2} \phi_{1} \varepsilon}{\beta_{4}}, \quad a_{22}=r_{2} K_{2} d-\delta-\beta_{2} r_{2} \bar{I}_{2}, \quad a_{32}=-\beta_{3} \bar{I}_{2}, \\
& a_{33}=-\frac{\left((1-k)^{2} \phi_{2} \varepsilon-\beta_{4}\left(K_{3}-\mu_{3}\right)\right)}{\beta_{4}}, \quad a_{34}=-(1-k) \phi_{2} \bar{I}_{2}, \quad a_{44}=-\beta_{4} .
\end{aligned}
$$

Theorem 4.2 Assume that $\phi_{1}>\frac{K_{1} r_{1} \beta_{4}}{\varepsilon(1-k)^{2}}$ and $\phi_{2}>\frac{\left(K_{3}-\delta\right) \beta_{4}}{\varepsilon(1-k)^{2}}$ holds. The dead critical point $\Lambda_{2}$ of system (3.1) is stable local asymptotic if and only if

$$
\bar{I}_{2}>\frac{\left(r_{2} K_{2} d-\delta\right) \beta_{4}}{(1-k)^{2} \phi_{1} \varepsilon},
$$

where $r_{2}>\frac{\delta}{K_{2} d}$.

Proof From (4.4), we obtain the following:

(i) $\lambda_{1}=r_{1} K_{1}-\frac{(1-k)^{2} \phi_{1} \varepsilon}{\beta_{4}}<0$ if $\phi_{1}>\frac{K_{1} r_{1} \beta_{4}}{\varepsilon(1-k)^{2}}$,

(ii) $\lambda_{2}=r_{2} K_{2} d-\delta-\beta_{2} r_{2} \bar{I}_{2}<0 \Rightarrow \bar{I}_{2}>\frac{\left(r_{2} K_{2} d-\delta\right) \beta_{4}}{(1-k)^{2} \phi_{1} \varepsilon}$, where $r_{2}>\frac{\delta}{K_{2} d}$,

(iii) $\lambda_{3}=-\frac{\left((1-k)^{2} \phi_{2} \varepsilon-\beta_{4}\left(K_{3}-\mu_{3}\right)\right)}{\beta_{4}}<0$, since $\phi_{2}>\frac{\left(K_{3}-\delta\right) \beta_{4}}{\varepsilon(1-k)^{2}}$,

(iv) $\lambda_{4}=-\beta_{4}<0$. 
Remark 4.2 In Theorem 4.2. it is shown that applying a low ketogenic diet increases the growth of the tumor population. In this scenario, the damage of DNA causes a malignant class in the breast tissues. The competition between the tumor cells and the immune system is intense, and the effect of the anti-cancer drug tamoxifen decreases the tumor population. However, the increase of the estrogen level also affected the healthy cell population to extinct as well.

Now, we consider the Jacobian matrix of dead critical point $2 ; \Lambda_{3}=\left(0, \bar{T}_{3}, \bar{I}_{3}, \frac{(1-k) \varepsilon}{\beta_{4}}\right)$, now we have

$$
J\left(\Lambda_{1}\right)=\left(\begin{array}{cccc}
a_{11} & 0 & 0 & 0 \\
a_{21} & a_{22} & a_{23} & 0 \\
0 & a_{32} & a_{33} & a_{34} \\
0 & 0 & 0 & a_{44}
\end{array}\right),
$$

where

$$
\begin{aligned}
& a_{11}=r_{1} K_{1}-\beta_{1} r_{1} \bar{T}_{3}-\frac{(1-k)^{2} \phi_{1} \varepsilon}{\beta_{4}}, \quad a_{21}=\frac{(1-k)^{2} \phi_{1} \varepsilon}{\beta_{4}} \bar{T}_{3}, \\
& a_{22}=\left(K_{2} d r_{2}-\delta\right)-2 r_{2} \mu_{2} \bar{T}_{3}-\beta_{2} r_{2} \bar{I}_{3}, \quad a_{23}=-\beta_{2} r_{2} \bar{I}_{3}, \quad a_{32}=-\beta_{3} \bar{I}_{3}, \\
& a_{33}=\left(K_{3}-\mu_{3}-\beta_{3} \bar{T}_{3}\right)-\frac{(1-k)^{2} \phi_{2} \varepsilon}{\beta_{4}}, \quad a_{34}=-(1-k) \phi_{2} \bar{I}_{3}, \quad a_{44}=-\beta_{4} .
\end{aligned}
$$

The characteristic equation of the dead critical point 2 is given by

$$
\left(a_{11}-\lambda\right)\left(a_{44}-\lambda\right)\left\{\left(a_{22}-\lambda\right)\left(a_{33}-\lambda\right)-a_{23} a_{32}\right\}=0 .
$$

Thus, the local stability for $\Lambda_{3}=\left(0, \bar{T}_{3}, \bar{I}_{3}, \bar{E}_{3}\right)$ is obtained in Theorem 4.3 as follows.

Theorem 4.3 Let $\Lambda_{3}$ be the critical point of system (3.1) and assume that $\phi_{1}>\frac{K_{1} r_{1} \beta_{4}}{\varepsilon(1-k)^{2}}$. Then the following statements are true:

(i) Let $r_{2}>\frac{\delta}{K_{2} d}$ and $R_{0}<1$. If

$$
\begin{aligned}
\bar{T}_{3} \in & \left(\frac{\left(K_{3}-\mu_{3}\right) \beta_{4}-(1-k)^{2} \phi_{2} \varepsilon}{\beta_{4} \beta_{3}}, \frac{K_{2} d r_{2}-\delta}{2 r_{2} \mu_{2}}\right), \\
\bar{I}_{3} \in & \left(\frac{\left\{\left(K_{2} d r_{2}+K_{3}\right) \beta_{4}-\left(\left(\mu_{3}+\delta\right) \beta_{4}+(1-k)^{2} \phi_{2} \varepsilon\right)\right\}-\left(2 r_{2} \mu_{2}+\beta_{3}\right) \beta_{4} \bar{T}_{3}}{\beta_{4} \beta_{2} r_{2}},\right. \\
& \left.\frac{\left(K_{2} d r_{2}-\delta\right)-2 r_{2} \mu_{2} \bar{T}_{3}}{\beta_{2} r_{2}}\right)
\end{aligned}
$$

and

$$
\bar{E}_{4} \in\left(\frac{2 r_{2} \mu_{2}\left(K_{3}-\mu_{3}\right)-\left(K_{2} d r_{2}-\delta\right) \beta_{3}}{2 r_{2} \mu_{2}(1-k) \phi_{2}}, \frac{\left(K_{2} d r_{2}+K_{3}-\left(\mu_{3}+\delta\right)\right)}{(1-k) \phi_{2}}\right)
$$

then both roots are real or complex conjugates with negative real parts and $\left|\arg \left(\lambda_{i}\right)\right|>\frac{\alpha \pi}{2}$ $(i=1,2,3,4)$ is equivalent to the Routh-Hurwitz criteria. This implies that $\Lambda_{3}$ is locally asymptotically stable. 
(ii) Let $r_{2}>\frac{\delta}{K_{2} d}$ and $R_{0}<1$. If

$$
\bar{T}_{3} \in\left(0, \frac{\left(K_{3}-\mu_{3}\right) \beta_{4}-(1-k)^{2} \phi_{2} \varepsilon}{\beta_{4} \beta_{3}}\right), \quad \bar{I}_{3} \in\left(\frac{\left(K_{2} d r_{2}-\delta\right)-2 r_{2} \mu_{2} \bar{T}_{3}}{\beta_{2} r_{2}}, \infty\right)
$$

and

$$
\bar{E}_{4} \in\left(\frac{2 r_{2} \mu_{2}\left(K_{3}-\mu_{3}\right)-\left(K_{2} d r_{2}-\delta\right) \beta_{3}}{2 r_{2} \mu_{2}(1-k) \phi_{2}}, \frac{\left(K_{2} d r_{2}+K_{3}-\left(\mu_{3}+\delta\right)\right)}{(1-k) \phi_{2}}\right),
$$

then both roots are complex conjugate with positive real parts and

$$
\begin{aligned}
\mid \tan ^{-1} & \left(-\left(4\left(\left(K_{2} d r_{2}-\delta\right)-2 r_{2} \mu_{2} \bar{T}_{3}-\beta_{2} r_{2} \bar{I}_{3}\right)\right.\right. \\
& \times\left(\left(K_{3}-\mu_{3}-\beta_{3} \bar{T}_{3}\right)-\frac{(1-k)^{2} \phi_{2} \varepsilon}{\beta_{4}}\right)\left(1-R_{0}\right) \\
& \left.-\left(\left(K_{2} d r_{2}-\delta\right)-2 r_{2} \mu_{2} \bar{T}_{3}-\beta_{2} r_{2} \bar{I}_{3}+\left(K_{3}-\mu_{3}-\beta_{3} \bar{T}_{3}\right)-\frac{(1-k)^{2} \phi_{2} \varepsilon}{\beta_{4}}\right)^{2}\right)^{\frac{1}{2}} \\
& \left.\times\left(\left(\left(K_{2} d r_{2}-\delta\right)-2 r_{2} \mu_{2} \bar{T}_{3}-\beta_{2} r_{2} \bar{I}_{3}+\left(K_{3}-\mu_{3}-\beta_{3} \bar{T}_{3}\right)-\frac{(1-k)^{2} \phi_{2} \varepsilon}{\beta_{4}}\right)\right)^{-1}\right) \mid \\
> & \frac{\alpha \pi}{2} .
\end{aligned}
$$

This implies that $\Lambda_{3}$ is locally asymptotically stable.

Proof From (4.7), we obtain

$$
\lambda_{1}=r_{1} K_{1}-\frac{(1-k)^{2} \phi_{1} \varepsilon}{\beta_{4}}<0 \quad \text { if } \phi_{1}>\frac{K_{1} r_{1} \beta_{4}}{\varepsilon(1-k)^{2}}
$$

and $\lambda_{4}=-\beta_{4}<0$. In this case, we need to consider only the characteristic equation

$$
\lambda^{2}-\left(a_{22}+a_{33}\right) \lambda+a_{22} a_{33}-a_{23} a_{32}=0 .
$$

Equation (4.9) shows the basic reproductive number, which is

$$
R_{0}=\frac{a_{23} a_{32}}{a_{22} a_{33}}
$$

for the characteristic equation

$$
\lambda^{2}-\left(a_{22}+a_{33}\right) \lambda+a_{22} a_{33}\left(1-\frac{a_{23} a_{32}}{a_{22} a_{33}}\right)=0
$$

which implies

$$
\lambda^{2}-\sum_{i=2}^{3} a_{i i} \lambda+\prod_{i=2}^{3} a_{i i}\left(1-R_{0}\right)=0
$$


(i) Let us consider the case where $\Delta=\left(a_{22}+a_{33}\right)^{2}-4 a_{22} a_{33}\left(1-R_{0}\right)>0$. For $R_{0}<1$ and $r_{2}>\frac{\delta}{K_{2} d}$, we have

$$
\begin{aligned}
& \frac{\left(K_{3}-\mu_{3}\right) \beta_{4}-(1-k)^{2} \phi_{2} \varepsilon}{\beta_{4} \beta_{3}}<\bar{T}_{3}<\frac{K_{2} d r_{2}-\delta}{2 r_{2} \mu_{2}} \text { and } \\
& \bar{I}_{3}<\frac{\left(K_{2} d r_{2}-\delta\right)-2 r_{2} \mu_{2} \bar{T}_{3}}{\beta_{2} r_{2}}
\end{aligned}
$$

which implies that $\Delta>0$. Moreover, computations show that

$$
\begin{aligned}
& a_{22}+a_{33}<0 \\
& \Rightarrow \quad \frac{\left\{\left(K_{2} d r_{2}+K_{3}\right) \beta_{4}-\left(\left(\mu_{3}+\delta\right) \beta_{4}+(1-k)^{2} \phi_{2} \varepsilon\right)\right\}-\left(2 r_{2} \mu_{2}+\beta_{3}\right) \beta_{4} \bar{T}_{3}}{\beta_{4} \beta_{2} r_{2}}<\bar{I}_{3},
\end{aligned}
$$

where

$$
\bar{T}_{3}<\frac{\left(K_{2} d r_{2}+K_{3}\right) \beta_{4}-\left(\left(\mu_{3}+\delta\right) \beta_{4}+(1-k)^{2} \phi_{2} \varepsilon\right)}{\left(2 r_{2} \mu_{2}+\beta_{3}\right) \beta_{4}}
$$

and

$$
\bar{E}_{4}<\frac{\left(K_{2} d r_{2}+K_{3}-\left(\mu_{3}+\delta\right)\right)}{(1-k) \phi_{2}}
$$

Considering (4.12)-(4.14), we obtain

$$
\begin{aligned}
& \frac{\left\{\left(K_{2} d r_{2}+K_{3}\right) \beta_{4}-\left(\left(\mu_{3}+\delta\right) \beta_{4}+(1-k)^{2} \phi_{2} \varepsilon\right)\right\}-\left(2 r_{2} \mu_{2}+\beta_{3}\right) \beta_{4} \bar{T}_{3}}{\beta_{4} \beta_{2} r_{2}} \\
& <\bar{I}_{3}<\frac{\left(K_{2} d r_{2}-\delta\right)-2 r_{2} \mu_{2} \bar{T}_{3}}{\beta_{2} r_{2}}
\end{aligned}
$$

and

$$
\begin{aligned}
& \frac{\left(K_{3}-\mu_{3}\right) \beta_{4}-(1-k)^{2} \phi_{2} \varepsilon}{\beta_{4} \beta_{3}} \\
& \quad<\bar{T}_{3}<\frac{K_{2} d r_{2}-\delta}{2 r_{2} \mu_{2}}<\frac{\left(K_{2} d r_{2}+K_{3}\right) \beta_{4}-\left(\left(\mu_{3}+\delta\right) \beta_{4}+(1-k)^{2} \phi_{2} \varepsilon\right)}{\left(2 r_{2} \mu_{2}+\beta_{3}\right) \beta_{4}},
\end{aligned}
$$

where

$$
\bar{E}_{4}>\frac{2 r_{2} \mu_{2}\left(K_{3}-\mu_{3}\right)-\left(K_{2} d r_{2}-\delta\right) \beta_{3}}{2 r_{2} \mu_{2}(1-k) \phi_{2}} .
$$

From (4.15) and (4.18), we get

$$
\frac{2 r_{2} \mu_{2}\left(K_{3}-\mu_{3}\right)-\left(K_{2} d r_{2}-\delta\right) \beta_{3}}{2 r_{2} \mu_{2}(1-k) \phi_{2}}<\bar{E}_{4}<\frac{\left(K_{2} d r_{2}+K_{3}-\left(\mu_{3}+\delta\right)\right)}{(1-k) \phi_{2}} \quad \text { for } r_{2}>\frac{\delta}{K_{2} d}
$$

(ii) Let us consider the case of complex roots with positive real parts. First of all, if

$$
a_{22}+a_{33}>0
$$


then we obtain positive real parts. Thus, for the inequality

$$
\begin{aligned}
& a_{22}+a_{33}>0 \\
& \Rightarrow \quad \frac{\left\{\left(K_{2} d r_{2}+K_{3}\right) \beta_{4}-\left(\left(\mu_{3}+\delta\right) \beta_{4}+(1-k)^{2} \phi_{2} \varepsilon\right)\right\}-\left(2 r_{2} \mu_{2}+\beta_{3}\right) \beta_{4} \bar{T}_{3}}{\beta_{4} \beta_{2} r_{2}}>\bar{I}_{3},
\end{aligned}
$$

where

$$
\bar{T}_{3}<\frac{\left(K_{2} d r_{2}+K_{3}\right) \beta_{4}-\left(\left(\mu_{3}+\delta\right) \beta_{4}+(1-k)^{2} \phi_{2} \varepsilon\right)}{\left(2 r_{2} \mu_{2}+\beta_{3}\right) \beta_{4}}
$$

and

$$
\bar{E}_{4}<\frac{\left(K_{2} d r_{2}+K_{3}-\left(\mu_{3}+\delta\right)\right)}{(1-k) \phi_{2}},
$$

we end up with (4.20).

Considering the condition $\Delta=\left(a_{22}+a_{33}\right)^{2}-4 a_{22} a_{33}\left(1-R_{0}\right)<0$, we have

$$
R_{0}<\frac{4 a_{22} a_{33}-\left(a_{22}+a_{33}\right)^{2}}{4 a_{22} a_{33}}<1 .
$$

Moreover, computations reveal that $4 a_{22} a_{33}>\left(a_{22}+a_{33}\right)^{2}$, if

$$
\bar{T}_{3}<\frac{\left(K_{3}-\mu_{3}\right) \beta_{4}-(1-k)^{2} \phi_{2} \varepsilon}{\beta_{4} \beta_{3}}
$$

and

$$
\bar{I}_{3}>\frac{\left(K_{2} d r_{2}-\delta\right)-2 r_{2} \mu_{2} \bar{T}_{3}}{\beta_{2} r_{2}},
$$

where

$$
\bar{T}_{3}<\frac{K_{2} d r_{2}-\delta}{2 r_{2} \mu_{2}}
$$

From (4.22), (4.25), and (4.27), we get

$$
\bar{T}_{3}<\frac{\left(K_{3}-\mu_{3}\right) \beta_{4}-(1-k)^{2} \phi_{2} \varepsilon}{\beta_{4} \beta_{3}}, \quad \bar{I}_{3}>\frac{\left(K_{2} d r_{2}-\delta\right)-2 r_{2} \mu_{2} \bar{T}_{3}}{\beta_{2} r_{2}}
$$

and

$$
\frac{2 r_{2} \mu_{2}\left(K_{3}-\mu_{3}\right)-\left(K_{2} d r_{2}-\delta\right) \beta_{3}}{2 r_{2} \mu_{2}(1-k) \phi_{2}}<\bar{E}_{4}<\frac{\left(K_{2} d r_{2}+K_{3}-\left(\mu_{3}+\delta\right)\right)}{(1-k) \phi_{2}} \quad \text { for } r_{2}>\frac{\delta}{K_{2} d}
$$

Remark 4.3 It is shown in Theorem 4.3 that the reproduction number has a dominant role to play in the dynamical system's stability. In this scenario, we assumed a weak immune response with low ketogenic support. It is seen that without the control parameters, the immune system is not strong enough to defend the tissues and the human body from the invasion of the malignant population. 
Let us consider the Jacobian matrix of the co-existing critical point: $\Lambda_{4}=\left(\bar{N}_{4}, \bar{T}_{4}, \bar{I}_{4}, \bar{E}_{4}\right)$, which has the form

$$
J\left(\Lambda_{4}\right)=\left(\begin{array}{cccc}
a_{11} & a_{12} & 0 & a_{14} \\
a_{21} & a_{22} & a_{23} & a_{24} \\
0 & a_{32} & a_{33} & a_{34} \\
0 & 0 & 0 & a_{44}
\end{array}\right)
$$

where

$$
\begin{aligned}
& a_{11}=r_{1} K_{1}-2 r_{1} \mu_{1} \bar{N}_{4}-r_{1} \beta_{1} \bar{T}_{4}-\frac{(1-k)^{2} \phi_{1} \varepsilon}{\beta_{4}}, \quad a_{12}=-\beta_{1} r_{1} \bar{N}_{4}, \\
& a_{14}=-(1-k) \phi_{1} \bar{N}_{4}, \quad a_{21}=\frac{(1-k)^{2} \phi_{1} \varepsilon \bar{T}_{4}}{\beta_{4}}, \\
& a_{22}=\left(K_{2} d r_{2}-\delta\right)-2 r_{2} \mu_{2} \bar{T}_{4}-\beta_{2} r_{2} \bar{I}_{4}+\frac{(1-k)^{2} \phi_{1} \varepsilon \bar{N}_{4}}{\beta_{4}}, \\
& a_{23}=-\beta_{2} r_{2} \bar{T}_{4}, \quad a_{24}=(1-k) \phi_{1} \bar{N}_{4} \bar{T}_{4}, \quad a_{32}=-\beta_{3} \bar{I}_{4}, \\
& a_{33}=\left(K_{3}-\mu_{3}-\beta_{3} \bar{T}_{4}\right)-\frac{(1-k)^{2} \phi_{2} \varepsilon}{\beta_{4}}, \\
& a_{34}=-(1-k) \phi_{2} \bar{I}_{4}, \quad a_{44}=-\beta_{4} .
\end{aligned}
$$

The characteristic equation of (4.30) around $\Lambda_{4}$ is given by

$$
\left(a_{11}-\lambda\right)\left\{\lambda^{2}-\left(a_{22}+a_{33}\right) \lambda+a_{22} a_{33}\left(1-R_{0}\right)\right\}-\left(a_{33}-\lambda\right) a_{12} a_{21}=0
$$

and

$$
\lambda_{4}=-\beta_{4}<0
$$

while (4.31) is a cubic equation of the form

$$
\begin{aligned}
\lambda^{3} & +\left(-a_{11}-a_{22}-a_{33}\right) \lambda^{2}+\left(a_{11} a_{22}+a_{11} a_{33}-a_{12} a_{21}+a_{23} a_{32}\left(1-R_{0}\right)\right) \lambda \\
& +\left(a_{33} a_{12} a_{21}-a_{11} a_{22} a_{33}\left(1-R_{0}\right)\right)=0 .
\end{aligned}
$$

Theorem 4.4 Let $\Lambda_{4}$ be the equilibrium point of system (3.1) and assume that $R_{0}<1$, $\phi_{1}<\frac{K_{1} r_{1} \beta_{4}}{\varepsilon(1-k)^{2}}$ and $\frac{\left(K_{3}-\delta\right) \beta_{4}}{\varepsilon(1-k)^{2}}<\phi_{2}<\frac{\left(K_{3}-\mu_{3}\right) \beta_{4}}{\varepsilon(1-k)^{2}}$. Then the following statements are true:

(i) Let $r_{1}>\frac{(1-k)^{2} \phi_{1} \varepsilon}{2 \beta_{4} \mu_{1}}$ and $r_{2}>\frac{\delta}{K_{2} d}$. If

$$
\bar{T}_{4}>\frac{r_{1} K_{1} \beta_{4}-(1-k)^{2} \phi_{1} \varepsilon}{\left(r_{1} \beta_{1}+2 r_{2} \mu_{2}\right) \beta_{4}} \quad \text { and } \quad \bar{I}_{4}>\frac{K_{2} d r_{2}-\delta}{\beta_{2} r_{2}}
$$

where $\beta_{3}>\frac{\left(r_{1} \beta_{1}+2 r_{2} \mu_{2}\right)\left(\left(K_{3}-\mu_{3}\right) \beta_{4}-(1-k)^{2} \phi_{2} \varepsilon\right)}{r_{1} K_{1} \beta_{4}-(1-k)^{2} \phi_{1} \varepsilon}$ then all the roots of $(4.33)$ are real. This implies that $\Lambda_{4}$ is locally asymptotically stable. 
(ii) Let $r_{1} \geq \frac{(1-k)^{2} \phi_{1} \varepsilon}{2 \beta_{4} \mu_{1}}$ and $r_{2}>\frac{\delta}{K_{2} d}$. If

$$
\begin{aligned}
& \bar{N}_{4} \in\left(\frac{\left(r_{1} K_{1}-r_{1} \beta_{1} \bar{T}_{4}\right) \beta_{4}-(1-k)^{2} \phi_{1} \varepsilon}{\beta_{4} r_{1}\left(2 \mu_{1}+\beta_{1}\right)}, \infty\right), \\
& \bar{T}_{4} \in\left[\frac{r_{1} K_{1} \beta_{4}-(1-k)^{2} \phi_{1} \varepsilon}{\left(r_{1} \beta_{1}+2 r_{2} \mu_{2}\right) \beta_{4}}, \frac{r_{1} K_{1} \beta_{4}-(1-k)^{2} \phi_{1} \varepsilon}{r_{1} \beta_{1} \beta_{4}}\right), \\
& \bar{I}_{4} \in\left[\frac{K_{2} d r_{2}-\delta}{\beta_{2} r_{2}}, \infty\right)
\end{aligned}
$$

and

$$
\left(\frac{(1-k) \phi_{1} \bar{E}_{4}+2 r_{2} \mu_{2}}{(1-k) \phi_{1} \bar{E}_{4}}\right)>\frac{\bar{N}_{4}}{\bar{T}_{4}}
$$

where $\beta_{3}=\frac{\left(r_{1} \beta_{1}+2 r_{2} \mu_{2}\right)\left(\left(K_{3}-\mu_{3}\right) \beta_{4}-(1-k)^{2} \phi_{2} \varepsilon\right)}{r_{1} K_{1} \beta_{4}-(1-k)^{2} \phi_{1} \varepsilon}$, then there is one real root and one complex root with its complex conjugate, which implies that $\Lambda_{4}$ is locally asymptotically stable.

Proof (i) If the discriminant of (4.33) is positive, then the Routh-Hurwitz conditions are necessary and sufficient conditions for locally asymptotically stability of $\Lambda_{4}$. Thus, we first consider the conditions for a positive discriminant of the cubic polynomial (4.33):

If

$$
\begin{aligned}
& a_{11}+a_{22}+a_{33}<0, \\
& a_{33} a_{12} a_{21}-a_{11} a_{22} a_{33}\left(1-R_{0}\right)>0, \\
& a_{11} a_{22}+a_{11} a_{33}-a_{12} a_{21}+a_{23} a_{32}\left(1-R_{0}\right)>0
\end{aligned}
$$

and

$$
\begin{aligned}
& \left(-a_{11}-a_{22}-a_{33}\right)\left(a_{11} a_{22}+a_{11} a_{33}-a_{12} a_{21}+a_{23} a_{32}\left(1-R_{0}\right)\right) \\
& \quad>a_{33} a_{12} a_{21}-a_{11} a_{22} a_{33}\left(1-R_{0}\right),
\end{aligned}
$$

then the discriminant of (4.33) is positive.

Because of (4.34), we have

$$
\begin{aligned}
& \left(\frac{(1-k)^{2} \phi_{1} \varepsilon}{\beta_{4}}-2 r_{1} \mu_{1}\right) \bar{N}_{4}+\left(r_{1} K_{1}-\left(r_{1} \beta_{1}+2 r_{2} \mu_{2}\right) \bar{T}_{4}-\frac{(1-k)^{2} \phi_{1} \varepsilon}{\beta_{4}}\right) \\
& +\left(K_{2} d r_{2}-\delta-\beta_{2} r_{2} \bar{I}_{4}\right)+\left(K_{3}-\mu_{3}-\frac{(1-k)^{2} \phi_{2} \varepsilon}{\beta_{4}}-\beta_{3} \bar{T}_{4}\right)<0,
\end{aligned}
$$

where we obtain

$$
\frac{(1-k)^{2} \phi_{1} \varepsilon}{\beta_{4}}-2 r_{1} \mu_{1}<0 \Rightarrow r_{1}>\frac{(1-k)^{2} \phi_{1} \varepsilon}{2 \beta_{4} \mu_{1}},
$$




$$
\begin{aligned}
& r_{1} K_{1}-\left(r_{1} \beta_{1}+2 r_{2} \mu_{2}\right) \bar{T}_{4}-\frac{(1-k)^{2} \phi_{1} \varepsilon}{\beta_{4}}<0 \\
& \Rightarrow \quad \bar{T}_{4}>\frac{r_{1} K_{1} \beta_{4}-(1-k)^{2} \phi_{1} \varepsilon}{\left(r_{1} \beta_{1}+2 r_{2} \mu_{2}\right) \beta_{4}} \text { for } \phi_{1}<\frac{K_{1} r_{1} \beta_{4}}{\varepsilon(1-k)^{2}}, \\
& K_{2} d r_{2}-\delta-\beta_{2} r_{2} \bar{I}_{4}<0 \Rightarrow \bar{I}_{4}>\frac{K_{2} d r_{2}-\delta}{\beta_{2} r_{2}} \quad \text { for } r_{2}>\frac{\delta}{K_{2} d}
\end{aligned}
$$

and

$$
\begin{aligned}
K_{3} & -\mu_{3}-\frac{(1-k)^{2} \phi_{2} \varepsilon}{\beta_{4}}-\beta_{3} \bar{T}_{4}<0 \\
\Rightarrow \quad \bar{T}_{4}>\frac{\left(K_{3}-\mu_{3}\right) \beta_{4}-(1-k)^{2} \phi_{2} \varepsilon}{\beta_{4} \beta_{3}} & \text { for } \phi_{2}<\frac{\left(K_{3}-\mu_{3}\right) \beta_{4}}{\varepsilon(1-k)^{2}} .
\end{aligned}
$$

Since $\Lambda_{4}$ is a positive critical point for $\phi_{2}>\frac{\left(K_{3}-\delta\right) \beta_{4}}{\varepsilon(1-k)^{2}}$, we see from (4.41) that $\mu_{3}<\delta$. Considering both (4.39) and (4.41), we have

$$
\bar{T}_{4}>\frac{r_{1} K_{1} \beta_{4}-(1-k)^{2} \phi_{1} \varepsilon}{\left(r_{1} \beta_{1}+2 r_{2} \mu_{2}\right) \beta_{4}}>\frac{\left(K_{3}-\mu_{3}\right) \beta_{4}-(1-k)^{2} \phi_{2} \varepsilon}{\beta_{4} \beta_{3}},
$$

where $\beta_{3}>\frac{\left(r_{1} \beta_{1}+2 r_{2} \mu_{2}\right)\left(\left(K_{3}-\mu_{3}\right) \beta_{4}-(1-k)^{2} \phi_{2} \varepsilon\right)}{r_{1} K_{1} \beta_{4}-(1-k)^{2} \phi_{1} \varepsilon}$.

On the other side, it is evident that (4.35) holds, since $a_{33}<0$. Moreover, considering (4.36) and (4.37), we obtain the result that it is satisfied with the conditions in (4.38)(4.42). This completes the proof of (i).

(ii) If the discriminant of (4.33) is negative, then there is only one real root and one complex root with its complex conjugate. Let us assume that

$$
\begin{aligned}
P(\lambda)= & (\lambda+a)(\lambda-b-i \omega)(\lambda-b+i \omega) \\
= & \lambda^{3}+\left(-a_{11}-a_{22}-a_{33}\right) \lambda^{2}+\left(a_{11} a_{22}+a_{11} a_{33}-a_{12} a_{21}+a_{23} a_{32}\left(1-R_{0}\right)\right) \lambda \\
& +\left(a_{33} a_{12} a_{21}-a_{11} a_{22} a_{33}\left(1-R_{0}\right)\right)=0 .
\end{aligned}
$$

Then

$$
\begin{aligned}
& a-2 b=-a_{11}-a_{22}-a_{33}, \\
& b^{2}+\omega^{2}-2 a b=a_{11} a_{22}+a_{11} a_{33}-a_{12} a_{21}+a_{23} a_{32}\left(1-R_{0}\right)
\end{aligned}
$$

and

$$
a\left(b^{2}+\omega^{2}\right)=a_{33} a_{12} a_{21}-a_{11} a_{22} a_{33}\left(1-R_{0}\right) .
$$

By the result in [36], since $a-2 b>0$ and $b \geq 0$,

$$
a_{11}+a_{22}+a_{33} \leq 0
$$

which holds for

$$
\frac{(1-k)^{2} \phi_{1} \varepsilon}{\beta_{4}}-2 r_{1} \mu_{1} \leq 0 \quad \Rightarrow \quad r_{1} \geq \frac{(1-k)^{2} \phi_{1} \varepsilon}{2 \beta_{4} \mu_{1}},
$$




$$
\begin{aligned}
& r_{1} K_{1}-\left(r_{1} \beta_{1}+2 r_{2} \mu_{2}\right) \bar{T}_{4}-\frac{(1-k)^{2} \phi_{1} \varepsilon}{\beta_{4}} \leq 0 \\
& \Rightarrow \quad \bar{T}_{4} \geq \frac{r_{1} K_{1} \beta_{4}-(1-k)^{2} \phi_{1} \varepsilon}{\left(r_{1} \beta_{1}+2 r_{2} \mu_{2}\right) \beta_{4}} \quad \text { for } \phi_{1}<\frac{K_{1} r_{1} \beta_{4}}{\varepsilon(1-k)^{2}}, \\
& K_{2} d r_{2}-\delta-\beta_{2} r_{2} \bar{I}_{4} \leq 0 \Rightarrow \bar{I}_{4} \geq \frac{K_{2} d r_{2}-\delta}{\beta_{2} r_{2}} \quad \text { for } r_{2}>\frac{\delta}{K_{2} d}
\end{aligned}
$$

and

$$
K_{3}-\mu_{3}-\frac{(1-k)^{2} \phi_{2} \varepsilon}{\beta_{4}}-\beta_{3} \bar{T}_{4} \leq 0 \quad \Rightarrow \quad \bar{T}_{4} \geq \frac{\left(K_{3}-\mu_{3}\right) \beta_{4}-(1-k)^{2} \phi_{2} \varepsilon}{\beta_{4} \beta_{3}}
$$

for $\frac{\left(K_{3}-\delta\right) \beta_{4}}{\varepsilon(1-k)^{2}}<\phi_{2}<\frac{\left(K_{3}-\mu_{3}\right) \beta_{4}}{\varepsilon(1-k)^{2}}$ where $\mu_{3}<\delta$. Considering both (4.46) and (4.48), we have

$$
\bar{T}_{4} \geq \frac{r_{1} K_{1} \beta_{4}-(1-k)^{2} \phi_{1} \varepsilon}{\left(r_{1} \beta_{1}+2 r_{2} \mu_{2}\right) \beta_{4}}
$$

where $\beta_{3}=\frac{\left(r_{1} \beta_{1}+2 r_{2} \mu_{2}\right)\left(\left(K_{3}-\mu_{3}\right) \beta_{4}-(1-k)^{2} \phi_{2} \varepsilon\right)}{r_{1} K_{1} \beta_{4}-(1-k)^{2} \phi_{1} \varepsilon}$.

On the other side, from [36] we have

$$
b^{2}+\omega^{2}-2 a b \geq 0 \quad \Rightarrow \quad b^{2}+\omega^{2} \geq 2 a b \quad \Rightarrow \quad b^{2} \sec ^{2} \theta \geq 2 a b \geq 4 b^{2},
$$

which implies that $|\arg (\lambda)|=\theta \geq \frac{\pi}{3}$ and from $|\arg (\lambda)|>\frac{\alpha \pi}{2}$, we have $\alpha<\frac{2}{3}$. Thus,

$$
a_{11} a_{22}+a_{11} a_{33}-a_{12} a_{21}+a_{23} a_{32}\left(1-R_{0}\right) \geq 0,
$$

which holds for the conditions in (4.45)-(4.49).

At last, we consider

$$
a_{33} a_{12} a_{21}-a_{11} a_{22} a_{33}\left(1-R_{0}\right)>0,
$$

which implies

$$
\begin{aligned}
\beta_{1} r_{1} \bar{N}_{4} \bar{T}_{4} \frac{(1-k)^{2} \phi_{1} \varepsilon}{\beta_{4}} & \\
> & -\left(r_{1} K_{1}-2 r_{1} \mu_{1} \bar{N}_{4}-r_{1} \beta_{1} \bar{T}_{4}-\frac{(1-k)^{2} \phi_{1} \varepsilon}{\beta_{4}}\right) \\
& \times\left(\left(K_{2} d r_{2}-\delta\right)-2 r_{2} \mu_{2} \bar{T}_{4}-\beta_{2} r_{2} \bar{I}_{4}+\frac{(1-k)^{2} \phi_{1} \varepsilon \bar{N}_{4}}{\beta_{4}}\right)\left(1-R_{0}\right)
\end{aligned}
$$

we have

$$
\bar{N}_{4}>\frac{\left(r_{1} K_{1}-r_{1} \beta_{1} \bar{T}_{4}\right) \beta_{4}-(1-k)^{2} \phi_{1} \varepsilon}{\beta_{4} r_{1}\left(2 \mu_{1}+\beta_{1}\right)},
$$

where

$$
\bar{T}_{4}<\frac{r_{1} K_{1} \beta_{4}-(1-k)^{2} \phi_{1} \varepsilon}{r_{1} \beta_{1} \beta_{4}}
$$


and

$$
\left(\frac{(1-k) \phi_{1} \bar{E}_{4}+2 r_{2} \mu_{2}}{(1-k) \phi_{1} \bar{E}_{4}}\right)>\frac{\bar{N}_{4}}{\bar{T}_{4}} .
$$

Remark 4.4 In Theorem 4.4, it is shown that, if the source rate of estrogen is low, then the interaction between the normal cells and tumor cells continues. At the same time, the growth of both populations has an inverse relation. According to the ketogenic assistance and supplements of an immune booster, a robust immune response is expected in this competition.

Example 4.1 In this example, the theoretical results are demonstrated by using numerical simulations. For this purpose, we wrote code and ran it using the MATLAB version 2019. The initial values of $(3.1)$ are $N(0)=200, T(0)=50, I(0)=50, E(0)=2$.

Figure 1 shows the limited competition between cancer and normal cell populations. After the cancer cells appear, some immune booster supplements support the normal cells in the interaction. The red graph is the cancer cell population $T(t)$, while the blue graph shows the normal cell population $N(t)$. It is seen that after a specific time, the tumor population becomes so strong that the immune system needs additional support from the control parameters. Therefore, to stabilize only the immune system is not sufficient for interaction against the malignant tumor.

In Figs. 2 and 3, we notice that introducing a ketogenic diet reduces the cancer cells. In this case, applying for the ketogenic program during the mixed-immunotherapy shows an effect on the per capita growth of the cancer cells and stabilizes the treatment to support the normal cells.

However, it is also known that increasing the rate of the ketogenic diet leads to ketoacidosis. Keto-acidosis is a composition of ketosis and acidosis. Ketosis is a substance known as ketone bodies, and acidosis is the acid of the blood, causing frequent polyuria, poor appetite, and loss of consciousness. Therefore, a rate at $d=0.3$ is reasonable, and the interaction can be supported by anti-cancer drugs such as tamoxifen. The tumor cell is given in red, while the normal cells are denoted in blue.

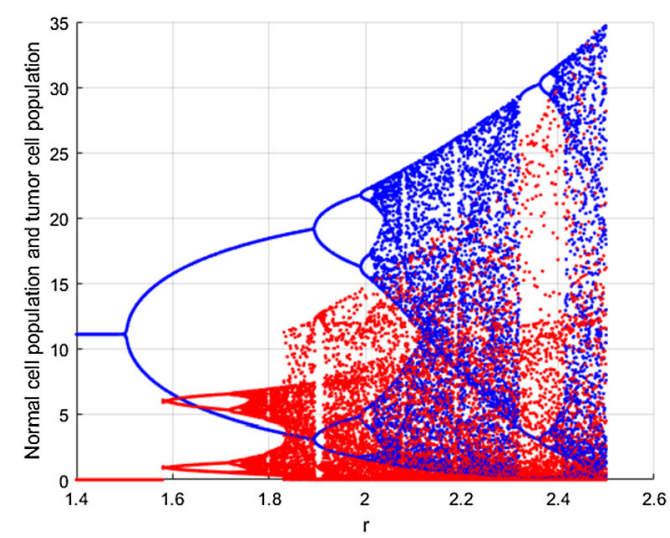

Figure 1 Bifurcation diagram of $T(t)$ and $N(t)$ with only one control parameter, the immune booster 


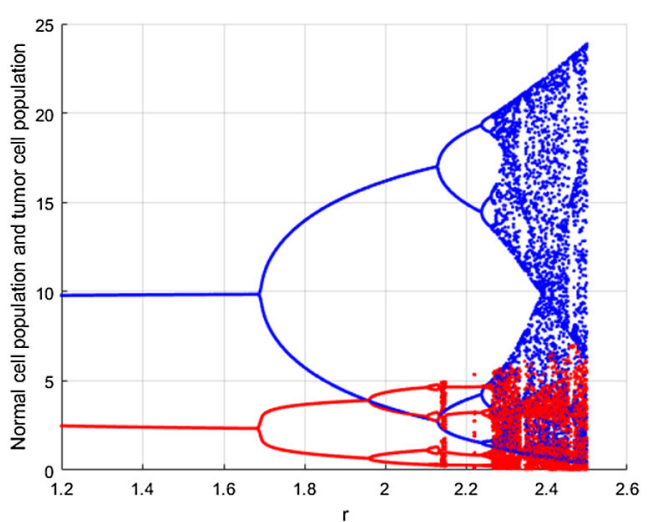

Figure 2 Bifurcation diagram of $T(t)$ and $N(t)$ with a mixed therapy including the ketogenic diet

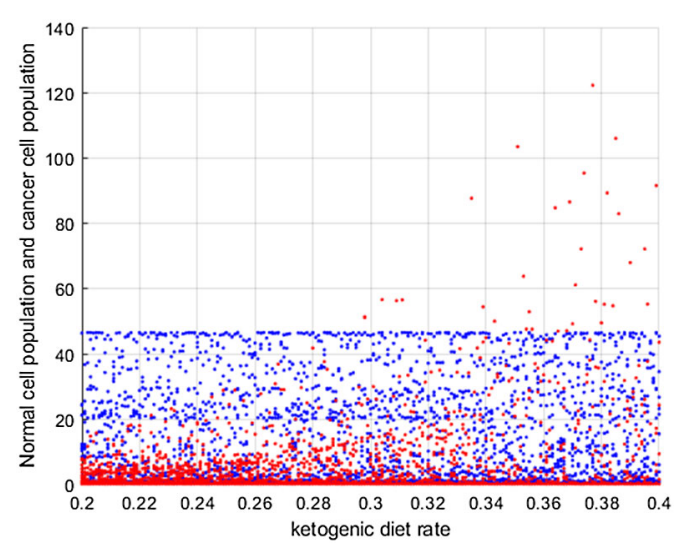

Figure 3 Effect of the ketogenic diet to the cell populations $T(t)$ and $N(t)$

\section{Existence and uniqueness}

Considering system (3.1) with the initial conditions $N(0)>0, T(0)>0, I(0)>0$ and $E(0)>$ 0 , the IVP can be written in matrix form as

$$
\left\{\begin{aligned}
& D^{\alpha} U(t)=M+A U(t)+N(t) B U(t)+T(t) C U(t) \\
&+I(t) D U(t)+E(t) G U(t), \quad t \in(0, T], \\
& U(0)=U_{0},
\end{aligned}\right.
$$

where $U(t)=\left[\begin{array}{c}N(t) \\ T(t) \\ I(t) \\ E(t)\end{array}\right]$ and $U(0)=\left[\begin{array}{c}N(0) \\ T(0) \\ I(0) \\ E(0)\end{array}\right]$ and $M=\left[\begin{array}{c}0 \\ 0 \\ \rho \gamma \\ (1-k) \varepsilon\end{array}\right]$.

Let us assume that $N(0) \leq v_{1}$, and $T(0)>0, I(0)>0, E(0)>0$, when $t>\sigma \geq 0$.

In this case, the following definitions can be applied to the main theorems in this section.

Definition 5.1 Let $C^{*}[0, T]$ be the class of continuous column vector $U(t)$ whose components $N(t), T(t), I(t), E(t) \in C[0, T]$ are the class of continuous functions on the interval 
$[0, T]$. The norm of $U \in C^{*}[0, T]$ is given by

$$
\|U\|=\sup _{t}\left|e^{-W t} N(t)\right|+\sup _{t}\left|e^{-W t} T(t)\right|+\sup _{t}\left|e^{-W t} I(t)\right|+\sup _{t}\left|e^{-W t} E(t)\right| .
$$

When $t>\sigma \geq 0$, we write $C_{\sigma}^{*}[0, T]$ and $C_{\sigma}[0, T]$.

Definition 5.2 Let the IVP of (5.1) have a solution given by $U \in C^{*}[0, T]$, if

(i) $(t, U(t)) \in D, t \in[0, T]$ where $D=[0, T] \times K$ and

$$
K=\left\{(N(t), T(t), I(t), E(t)):|N(t)| \leq v_{1},|T(t)| \leq v_{2},|I(t)| \leq v_{3},|E(t)| \leq v_{4}\right\} .
$$

(ii) $U(t)$ satisfies (5.1).

Theorem 5.1 The IVP of (5.1) has a unique solution $U \in C^{*}[0, T]$.

Proof Let us write

$$
I^{1-\alpha} \frac{d}{d t} U(t)=M+A U(t)+N(t) B U(t)+T(t) C U(t)+I(t) D U(t)+E(t) G U(t) .
$$

Operating with $I^{\alpha}$, we obtain

$$
U(t)=U(0)+I^{\alpha}(M+A U(t)+N(t) B U(t)+T(t) C U(t)+I(t) D U(t)+E(t) G U(t)) .
$$

Now let $F: C^{*}[0, T] \rightarrow C^{*}[0, T]$ be defined by

$$
F U(t)=U(0)+I^{\alpha}(M+A U(t)+N(t) B U(t)+T(t) C U(t)+I(t) D U(t)+E(t) G U(t)) .
$$

Then we get

$$
\begin{aligned}
e^{-W t} & \|F U-F V\| \\
= & e^{-W t} I^{\alpha}(A(U(t)-V(t))+N(t) B(U(t)-V(t))+T(t) C(U(t)-V(t)) \\
& +I(t) D(U(t)-V(t))+E(t) G(U(t)-V(t))) \\
\leq & \frac{1}{\Gamma(\alpha)} \int_{0}^{t}(t-s)^{\alpha-1} e^{-W(t-s)}(U(s)-V(s)) e^{-W /}\left(A+v_{1} B+v_{2} C+v_{3} D+v_{4} G\right) d s \\
\leq & \frac{\left(A+v_{1} B+v_{2} C+v_{3} D+v_{4} G\right)}{W^{\alpha}}\|U-V\| \int_{0}^{t} \frac{s^{\alpha-1}}{\Gamma(\alpha)} d s .
\end{aligned}
$$

This implies that $\|F U-F V\| \leq \frac{\left(A+v_{1} B+v_{2} C+v_{3} D+v_{4} G\right)}{W^{\alpha}}\|U-V\|$. If we choose W such that $W^{\alpha}>A+v_{1} B+v_{2} C+v_{3} D+v_{4} G$, then we obtain $\|F U-F V\| \leq\|U-V\|$. Moreover, the operator $F$ given by (4.4) has a unique fixed point.

Consequently, (5.3) has a unique solution $U \in C^{*}[0, T]$. From (5.3), we have

$$
\begin{aligned}
U(t)= & U(0)+\left(\frac{t^{\alpha}}{\Gamma(\alpha+1)}(M+A U(t)+N(t) B U(t)+T(t) C U(t)\right. \\
& +I(t) D U(t)+E(t) G U(t)))
\end{aligned}
$$




$$
\begin{aligned}
& +I^{\alpha+1}\left(A U^{\prime}(t)+N^{\prime}(t) B U(t)+N(t) B U^{\prime}(t)+T^{\prime}(t) C U(t)+T(t) C U^{\prime}(t)\right. \\
& \left.+I^{\prime}(t) D U(t)+I(t) D U^{\prime}(t)+E^{\prime}(t) G U(t)+E(t) G U^{\prime}(t)\right)
\end{aligned}
$$

and

$$
\begin{aligned}
\frac{U(t)}{d t}= & \frac{t^{\alpha-1}}{\Gamma(\alpha)}(M+A U(0)+N(0) B U(0)+T(0) C U(0)+I(0) D U(0)+E(0) G U(0)) \\
& +I^{\alpha}\left(A U^{\prime}(t)+N^{\prime}(t) B U(t)+N(t) B U^{\prime}(t)+T^{\prime}(t) C U(t)+T(t) C U^{\prime}(t)\right. \\
& \left.+I^{\prime}(t) D U(t)+I(t) D U^{\prime}(t)+E^{\prime}(t) G U(t)+E(t) G U^{\prime}(t)\right),
\end{aligned}
$$

which implies

$$
\begin{aligned}
e^{-N t}( & \left.\frac{U(t)}{d t}\right) \\
= & e^{-N t}\left(\frac{t^{\alpha-1}}{\Gamma(\alpha)}(M+A U(0)+N(0) B U(0)+T(0) C U(0)+I(0) D U(0)+E(0) G U(0))\right. \\
& +I^{\alpha}\left(A U^{\prime}(t)+N^{\prime}(t) B U(t)+N(t) B U^{\prime}(t)+T^{\prime}(t) C U(t)+T(t) C U^{\prime}(t)\right. \\
& \left.\left.+I^{\prime}(t) D U(t)+I(t) D U^{\prime}(t)+E^{\prime}(t) G U(t)+E(t) G U^{\prime}(t)\right)\right)
\end{aligned}
$$

from which we can deduce that $U^{\prime} \in C_{\sigma}^{*}[0, T]$. Thus, we have

$$
\frac{d U(t)}{d t}=\frac{d}{d t} I^{\alpha}(M+A U(t)+N(t) B U(t)+T(t) C U(t)+I(t) D U(t)+E(t) G U(t)),
$$

which implies

$$
I^{1-\alpha} \frac{d U(t)}{d t}=I^{1-\alpha} \frac{d}{d t} I^{\alpha}(M+A U(t)+N(t) B U(t)+T(t) C U(t)+I(t) D U(t)+E(t) G U(t))
$$

or

$$
D^{\alpha} U(t)=M+A U(t)+N(t) B U(t)+T(t) C U(t)+I(t) D U(t)+E(t) G U(t)
$$

and

$$
U(0)=U_{0}+I^{\alpha}(M+A U(0)+N(0) B U(0)+T(0) C U(0)+I(0) D U(0)+E(0) G U(0))=U_{0} .
$$

Therefore, this IVP is equivalent to (5.1), which completes the proof.

\section{An analysis of the tumor growth at low density}

In 1838, Verhulst considered the logistic growth function to explain mono-species growth. Later on, it was demonstrated that the logistic equation needs modifications to explain the growth of the population in low density-size, which is known as the Allee effect.

The Allee effect can be divided into two main types:

- strong Allee effect and

- weak Allee effect. 
A population with a strong Allee effect will have a critical population size, the population's threshold. Any size that is less than the threshold will go to extinction without any further aid. However, a population with a weak Allee effect will reduce the per capita growth rate at a lower population density or size [37-41].

Based on the above discussion, we apply the weak Allee function at time $t$ to the system (3.1), such as

$$
\left\{\begin{array}{l}
D^{\alpha} N(t)=N(t) r_{1}\left(K_{1}-\mu_{1} N(t)-\beta_{1} T(t)\right)-(1-k) \phi_{1} N(t) E(t) \\
D^{\alpha} T(t)=a(T(t)) \cdot T(t)\left\{r_{2}\left(K_{2} d-\mu_{2} T(t)-\beta_{2} I(t)\right)-\delta+(1-k) \phi_{1} N(t) E(t)\right\} \\
D^{\alpha} I(t)=\rho \gamma+I(t)\left(K_{3}-\mu_{3}-\beta_{3} T(t)\right)-(1-k) \phi_{2} I(t) E(t) \\
D^{\alpha} E(t)=(1-k) \varepsilon-\beta_{4} E(t)
\end{array}\right.
$$

where $t>0$ and $(N(0), T(0), I(0), E(0))=\left(N_{0}, T_{0}, I_{0}, E_{0}\right)$. Moreover, we define $\varpi>0$ as the Allee coefficient of the tumor population and $a(T(t))=\frac{T(t)}{\omega+T(t)}$ is the Allee function.

Considering the density of class $T(t)$, we can see that the Allee function is useful for the malignant class if

$$
T(t) \in\left(0,-\varpi+\sqrt{\frac{\varpi\left(\mu_{2} r_{2} \varpi+K_{2} d r_{2}-\delta-\beta_{2} r_{2} I(t)+(1-k) \phi_{1} N(t) E(t)\right)}{\mu_{2} r_{2}}}\right),
$$

where the immune system is also dependent on the Allee coefficient,

$$
I(t)<\frac{\mu_{2} r_{2} \varpi+K_{2} d r_{2}-\delta}{\beta_{2} r_{2}} \text { for } r_{2}>\frac{\delta}{K_{2} d}
$$

For a low population size of the tumor population, let us consider the stability conditions around $\Lambda_{4}$. The characteristic equation is given by

$$
\left(a_{11}-\lambda\right)\left\{\lambda^{2}-\left(a_{22}+a_{33}\right) \lambda+a_{22} a_{33}\left(1-R_{0}\right)\right\}-\left(a_{33}-\lambda\right) a_{12} a_{21}=0
$$

and

$$
\lambda_{4}=-\beta_{4}<0
$$

while (6.4) is a cubic equation of the form

$$
\begin{aligned}
& \lambda^{3}+\left(-a_{11}-a_{22}-a_{33}\right) \lambda^{2}+\left(a_{11} a_{22}+a_{11} a_{33}-a_{12} a_{21}+a_{23} a_{32}\left(1-R_{0}\right)\right) \lambda \\
& \quad+\left(a_{33} a_{12} a_{21}-a_{11} a_{22} a_{33}\left(1-R_{0}\right)\right)=0
\end{aligned}
$$

and

$$
\begin{aligned}
& a_{11}=r_{1} K_{1}-2 r_{1} \mu_{1} \bar{N}_{4}-r_{1} \beta_{1} \bar{T}_{4}-\frac{(1-k)^{2} \phi_{1} \varepsilon}{\beta_{4}}, \quad a_{12}=-\beta_{1} r_{1} \bar{N}_{4}, \\
& a_{14}=-(1-k) \phi_{1} \bar{N}_{4}, \quad a_{21}=\frac{(1-k)^{2} \phi_{1} \varepsilon \bar{T}_{4} a\left(\bar{T}_{4}\right)}{\beta_{4}}, \\
& a_{22}=a\left(\bar{T}_{4}\right)\left(\left(K_{2} d r_{2}-\delta\right)-2 r_{2} \mu_{2} \bar{T}_{4}-\beta_{2} r_{2} \bar{I}_{4}+\frac{(1-k)^{2} \phi_{1} \varepsilon \bar{N}_{4}}{\beta_{4}}\right),
\end{aligned}
$$




$$
\begin{aligned}
& a_{23}=-\beta_{2} r_{2} \bar{T}_{4} a\left(\bar{T}_{4}\right), \quad a_{24}=(1-k) \phi_{1} \bar{N}_{4} \bar{T}_{4} a\left(\bar{T}_{4}\right), \quad a_{32}=-\beta_{3} \bar{I}_{4}, \\
& a_{33}=\left(K_{3}-\mu_{3}-\beta_{3} \bar{T}_{4}\right)-\frac{(1-k)^{2} \phi_{2} \varepsilon}{\beta_{4}}, \quad a_{34}=-(1-k) \phi_{2} \bar{I}_{4}, \quad a_{44}=-\beta_{4} .
\end{aligned}
$$

Theorem 6.1 Let $\Lambda_{4}$ be the positive equilibrium point of system (6.1) and assume that $R_{0}<1, \phi_{1}<\frac{K_{1} r_{1} \beta_{4}}{\varepsilon(1-k)^{2}}$ and $\frac{\left(K_{3}-\delta\right) \beta_{4}}{\varepsilon(1-k)^{2}}<\phi_{2}<\frac{\left(K_{3}-\mu_{3}\right) \beta_{4}}{\varepsilon(1-k)^{2}}$. Then the following statements are true:

(i) Let $r_{1}>\frac{(1-k)^{2} \phi_{1} \varepsilon a\left(\bar{T}_{4}\right)}{2 \beta_{4} \mu_{1}}$ and $r_{2}>\frac{\delta}{K_{2} d}$. If

$$
\bar{T}_{4}>\frac{r_{1} K_{1} \beta_{4}-(1-k)^{2} \phi_{1} \varepsilon}{\left(r_{1} \beta_{1}+2 r_{2} \mu_{2} a\left(\bar{T}_{4}\right)\right) \beta_{4}} \quad \text { and } \quad \bar{I}_{4}>\frac{K_{2} d r_{2}-\delta}{\beta_{2} r_{2}}
$$

where $\beta_{3}>\frac{\left(r_{1} \beta_{1}+2 r_{2} \mu_{2} a\left(\bar{T}_{4}\right)\right)\left(\left(K_{3}-\mu_{3}\right) \beta_{4}-(1-k)^{2} \phi_{2} \varepsilon\right)}{r_{1} K_{1} \beta_{4}-(1-k)^{2} \phi_{1} \varepsilon}$ then all the roots are real. This implies that $\Lambda_{4}$ is locally asymptotically stable.

(ii) Let $r_{1} \geq \frac{(1-k)^{2} \phi_{1} \varepsilon a\left(\bar{T}_{4}\right)}{2 \beta_{4} \mu_{1}}$ and $r_{2}>\frac{\delta}{K_{2} d}$. If

$$
\begin{aligned}
& \bar{N}_{4} \in\left(\frac{\left(r_{1} K_{1}-r_{1} \beta_{1} \bar{T}_{4}\right) \beta_{4}-(1-k)^{2} \phi_{1} \varepsilon}{\beta_{4} r_{1}\left(2 \mu_{1}+\beta_{1}\right)}, \infty\right), \\
& \bar{T}_{4} \in\left[\frac{r_{1} K_{1} \beta_{4}-(1-k)^{2} \phi_{1} \varepsilon}{\left(r_{1} \beta_{1}+2 r_{2} \mu_{2} a\left(\bar{T}_{4}\right)\right) \beta_{4}}, \frac{r_{1} K_{1} \beta_{4}-(1-k)^{2} \phi_{1} \varepsilon}{r_{1} \beta_{1} \beta_{4}}\right), \\
& \bar{I}_{4} \in\left[\frac{K_{2} d r_{2}-\delta}{\beta_{2} r_{2}}, \infty\right)
\end{aligned}
$$

and

$$
\left(\frac{(1-k) \phi_{1} \bar{E}_{4}+2 r_{2} \mu_{2} a\left(\bar{T}_{4}\right)}{(1-k) \phi_{1} \bar{E}_{4}}\right)>\frac{\bar{N}_{4}}{\bar{T}_{4}}
$$

where $\beta_{3}=\frac{\left(r_{1} \beta_{1}+2 r_{2} \mu_{2} a\left(\bar{T}_{4}\right)\right)\left(\left(K_{3}-\mu_{3}\right) \beta_{4}-(1-k)^{2} \phi_{2} \varepsilon\right)}{r_{1} K_{1} \beta_{4}-(1-k)^{2} \phi_{1} \varepsilon}$, then there are only one real root and one complex root with its complex conjugate, which imply that $\Lambda_{4}$ is locally asymptotically stable.

Proof (i) Let us consider the conditions for a positive discriminant of (6.6). Thus,

$$
\begin{aligned}
& +\left(\frac{(1-k)^{2} \phi_{1} \varepsilon a\left(\bar{T}_{4}\right)}{\beta_{4}}-2 r_{1} \mu_{1}\right) \bar{N}_{4}+\left(r_{1} K_{1}-\left(r_{1} \beta_{1}+2 r_{2} \mu_{2} a\left(\bar{T}_{4}\right)\right) \bar{T}_{4}-\frac{(1-k)^{2} \phi_{1} \varepsilon}{\beta_{4}}\right) \\
& \quad+a\left(\bar{T}_{4}\right)\left(K_{2} d r_{2}-\delta-\beta_{2} r_{2} \bar{I}_{4}\right)+\left(K_{3}-\mu_{3}-\beta_{3} \bar{T}_{4}-\frac{(1-k)^{2} \phi_{2} \varepsilon}{\beta_{4}}\right)<0
\end{aligned}
$$

where we obtain

$$
\begin{gathered}
\frac{(1-k)^{2} \phi_{1} \varepsilon a\left(\bar{T}_{4}\right)}{\beta_{4}}-2 r_{1} \mu_{1}<0 \Rightarrow r_{1}>\frac{(1-k)^{2} \phi_{1} \varepsilon a\left(\bar{T}_{4}\right)}{2 \beta_{4} \mu_{1}}, \\
r_{1} K_{1}-\left(r_{1} \beta_{1}+2 r_{2} \mu_{2} a\left(\bar{T}_{4}\right)\right) \bar{T}_{4}-\frac{(1-k)^{2} \phi_{1} \varepsilon}{\beta_{4}}<0 \\
\Rightarrow \quad \bar{T}_{4}>\frac{r_{1} K_{1} \beta_{4}-(1-k)^{2} \phi_{1} \varepsilon}{\left(r_{1} \beta_{1}+2 r_{2} \mu_{2} a\left(\bar{T}_{4}\right)\right) \beta_{4}} \quad \text { for } \phi_{1}<\frac{K_{1} r_{1} \beta_{4}}{\varepsilon(1-k)^{2}},
\end{gathered}
$$




$$
K_{2} d r_{2}-\delta-\beta_{2} r_{2} \bar{I}_{4}<0 \quad \Rightarrow \quad \bar{I}_{4}>\frac{K_{2} d r_{2}-\delta}{\beta_{2} r_{2}} \text { for } r_{2}>\frac{\delta}{K_{2} d}
$$

and

$$
\begin{aligned}
K_{3} & -\mu_{3}-\frac{(1-k)^{2} \phi_{2} \varepsilon}{\beta_{4}}-\beta_{3} \bar{T}_{4}<0 \\
\Rightarrow \quad T_{4}>\frac{\left(K_{3}-\mu_{3}\right) \beta_{4}-(1-k)^{2} \phi_{2} \varepsilon}{\beta_{4} \beta_{3}} & \text { for } \phi_{2}<\frac{\left(K_{3}-\mu_{3}\right) \beta_{4}}{\varepsilon(1-k)^{2}} .
\end{aligned}
$$

Considering both (6.9) and (6.11), we have

$$
\bar{T}_{4}>\frac{r_{1} K_{1} \beta_{4}-(1-k)^{2} \phi_{1} \varepsilon}{\left(r_{1} \beta_{1}+2 r_{2} \mu_{2} a\left(\bar{T}_{4}\right)\right) \beta_{4}}>\frac{\left(K_{3}-\mu_{3}\right) \beta_{4}-(1-k)^{2} \phi_{2} \varepsilon}{\beta_{4} \beta_{3}},
$$

where $\beta_{3}>\frac{\left(r_{1} \beta_{1}+2 r_{2} \mu_{2} a\left(\bar{T}_{4}\right)\right)\left(\left(K_{3}-\mu_{3}\right) \beta_{4}-(1-k)^{2} \phi_{2} \varepsilon\right)}{r_{1} K_{1} \beta_{4}-(1-k)^{2} \phi_{1} \varepsilon}$. This completes the proof of (i).

Since Theorem $4.4 /$ (ii) is similar to (i), it is given without proof.

Remark 6.1 Theorem 6.1 shows that the ketogenic diet affects the growth rate of the tumor population. Decreasing the ketogenic rate is increasing the tumor growth rate, which leads to a weak immune response. On the other side, increasing the ketogenic program in continuous time restricts the tumor population's capacity, supporting the immune system. Additionally, we noticed that other complement control parameters such as anti-cancer drugs decrease the estrogen level caused by damage to the DNA.

Example 6.1 In this example, we wrote code and ran it using the MATLAB version 2019. The initial values of $(3.1)$ are $N(0)=200, T(0)=50, I(0)=50, E(0)=2$. Figure 4 shows the graph of the tumor cells and the normal cells, where $T(t)$ is given in red and $N(t)$ in blue for mixed therapy in the case of early detection. All control parameters are included in the treatment with low dosages, which drive the tumor density extinct in a short discrete time. This simulation demonstrates the weak Allee effect, where the Allee coefficient is $\varpi=0.4$. Therefore, it is seen that the ketogenic assistance and supplements of an immune booster are essential in all stages of cancer treatment.

\section{Conclusions}

This study analyzed a mathematical model of breast cancer as a fractional-order system to analyze tumor growth under chemotherapeutic treatment and immune response. We incorporated sensitive control parameters (ketogenic diet, immune booster, and anti-cancer drugs). Further, we assumed in the model that the majority of the malignant cells are ESR1 + ve.

In Sect. 2, we established the model, and in Sect. 3, we obtained four essential equilibrium points: the tumor-free equilibrium point, the death equilibrium point 1-2, and the co-existing equilibrium point. We proved in Sect. 4 that if the tumor cells' invasion of the healthy cells is small, then the malignant tumor size can be eliminated from the breast tissues because of the immune response and estrogen level seen for the tumor-free equilibrium point. We found that the ketogenic diet rate supports the immune response to fight against tumor growth but cannot stop the estrogen level for the death equilibrium points. For the co-existing positive equilibrium point, it is shown that if we have a low rate of the 


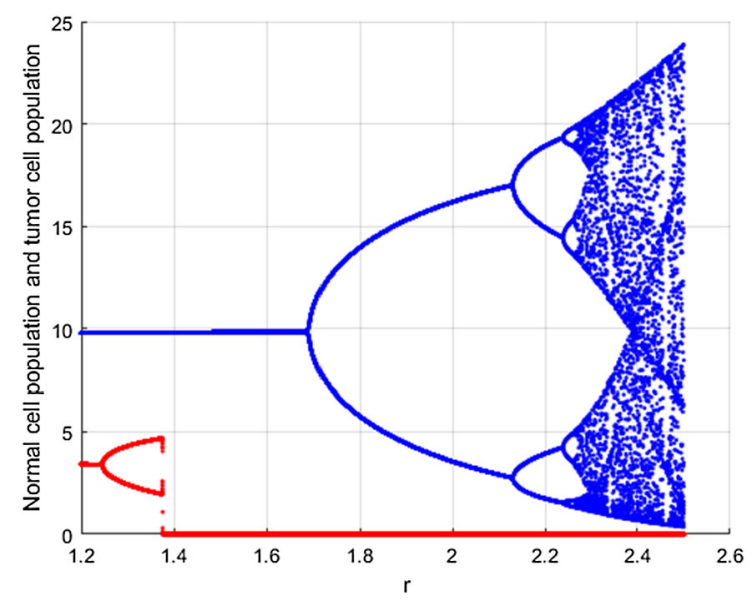

Figure 4 Bifurcation diagram of $T(t)$ and $N(t)$ with a mixed therapy with Allee effect

estrogen level, then the normal cells and the cancer cells are interactional stables. This occurs with a robust immune system relevant to the support of ketogenic assistance and the immune booster's supplements. After that, we considered the existence and uniqueness of the initial value problem in Sect. 5.

Finally, in Sect. 6, we considered the early detection case of the tumor population by applying the weak Allee effect at a discrete-time of $t$. We show that the Allee coefficient affects the normal cell population growth. We emphasized that the DNA damage caused by the estrogen level is still low in the early stages of the malignant population. The essential point was that the ketogenic diet rate was not related to the malignant class density, while the immune response was concerning the tumor growth and the Allee coefficient.

\section{Acknowledgements}

The Scientific Research Council supports this work at Erciyes University with the project code FBA-2014-5336.

\section{Funding}

There is no financial support from any private or public organization. There is no financial relationship with any individuals (friends, spouse, relatives, ...).

\section{Availability of data and materials}

All data generated or analyzed during this study are included in this published article.

Competing interests

The authors declare that they have no competing interests.

Authors' contributions

FB and AY conceived of the study and were in charge of overall direction and planning. They designed the model and set up the main parts of the study. All authors set up the theorems and proved them. FB did the simulation results using Matlab 2019. The authors wrote the manuscript and revised it to the submitted form. There is no ghost-writing. All authors read and approved the final manuscript.

\section{Author details}

'Department of Mathematics, Kuwait College of Science and Technology, 27235 Kuwait City, Kuwait. ${ }^{2}$ Department of Mathematics, Faculty of Education, Erciyes University, 38039 Kayseri, Turkey. ${ }^{3}$ Department of Mathematics and General Sciences, Prince Sultan University, 11586, Riyadh, Saudi Arabia. ${ }^{4}$ Department of Medical Research, China Medical University, Taichung 40402, Taiwan.

\section{Publisher's Note}

Springer Nature remains neutral with regard to jurisdictional claims in published maps and institutional affiliations. 


\section{References}

1. El-Gohary, A.: Chaos and optimal control of cancer self-remission and tumor system steady states. Chaos Solitons Fractals 37, 1305-1316 (2008)

2. World Health Organization: Organization, Global Action Plan for the Prevention and Control on NCDs. World Health Organization, Geneva (2014)

3. Oke, S.I., Matadi, M.B., Xulu, S.S.: Optimal control analysis of a mathematical model for breast cancer. Math. Comput. Appl. 23, 21 (2018)

4. Olayebi, O.O., Agbobu, S.C., Wauton, I., Olufemi, A.S.: Mathematical modelling of breast cancer thermo-therapy treatment: ultrasound-based approach. J. Multidiscip. Eng. Sci. Stud. 2(12), 1158-1164 (2016)

5. Patel, M.I., Nagl, S.: The Role of the Model Integration in Complex Systems Modeling: An Example of Cancer Biology. Springer, Berlin (2010)

6. Sethi, M., Chakravarti, S.K.: Hyperthermia techniques for cancer treatment: a review. Int. J. PharmTech Res. 8(6), 292-299 (2015)

7. Wei, H.C.: Mathematical modeling of ER-positive breast cancer treatment with AZD9496 and palbociclib. AIMS Math. 5(4), 3446-3455 (2020)

8. Botasteanu, D.A., Lipkuwitz, S., Lee, J.M., Levy, D.: Mathematical models of breast and ovarian cancers. Wiley Interdiscip. Rev., Syst. Biol. Med. 8(4), 337-362 (2016)

9. Dey, S.K., Charlies, S.: Mathematical model of breast cancer treatment. Springer Proc. Math. Stat. 146, 149-160 (2015)

10. Solis-Perez, J.E., Gomez-Agilar, J.F., Atangana, A.: A fractional mathematical model of breast cancer competition model. Chaos Solitons Fractals 127, 38-54 (2019)

11. Valentim, C.A. Jr., Oliveira, N.A., Rabi, J.A., David, S.A.: Can fractional calculus help improve tumor growth models? J. Comput. Appl. Math. 379, 1-15 (2020)

12. Sweilam, N.H., Al-Mekhlafi, S.M., Assiri, T., Atangana, A.: Optimal control for cancer treatment mathematical model using Atangana-Baleanu-Caputo fractional derivative. Adv. Differ. Equ. 2020, 334 (2020)

13. Huang, C., Xiao, M., Alsaedi, A., Hayat, T.: Bifurcations in a delayed fractional complex-valued neural network. Appl. Math. Comput. 292, 210-227 (2017)

14. Huang, C., Cao, J., Xiao, M., Alsaedi, A., Alsaadi, F.E.: Controlling bifurcation in a delayed fractional predator-prey system with incommensurate orders. Appl. Math. Comput. 293, 293-310 (2017)

15. Huang, C., Cao, J.D., Xiao, M., Alsaedi, A., Hayat, T.: Effects of time delays on stability and Hopf bifurcation in a fractional-order ring-structured network with arbitrary neurons. Commun. Nonlinear Sci. Numer. Simul. 57, 1-13 (2018)

16. Solis-Perez, J.E., Gomez-Aguilar, J.F.: Variable-order fractal-fractional time delay equations with power, exponential and Mittag-Leffler laws and their numerical solutions. Eng. Comput. 1, 1-23 (2020)

17. Atangana, A., Gomez-Aguilar, J.F.: Numerical approximation of Riemann-Liouville definition of fractional derivative: from Riemann-Liouville to Atangana-Baleanu. Numer. Methods Partial Differ. Equ. 34(5), 1502-1523 (2017)

18. Yousef, A., Bozkurt, F.: Bifurcation and stability analysis of a system of fractional-order differential equations for a plant-herbivore model with Allee effect. Mathematics 7(5), 454 (2019)

19. Al-Khaled, K., Alquran, M.: An approximate solution for a fractional-order model of generalized Harry Dym equation. Math. Sci. 8, 125-130 (2014)

20. Ahmad, W.M., Sprott, J.C.: Chaos in fractional order autonomous nonlinear systems. Chaos Solitons Fractals 16 , 339-351 (2003)

21. d'Ornafrio, A., Ledzewicz, U., Maurer, H., Schaettler, H.: On optimal delivery of combination therapy for tumors. Math Biosci. 222, 13-26 (2009)

22. Kermarck, W.O., Mc Kendrick, A.G.: Contributions to the mathematical theory of epidemics. Proc. R. Soc. Lond. Ser. A $115,700-721(1927)$

23. Kermarck, W.O., Mc Kendrick, A.G.: Contributions to the mathematical theory of epidemics. V. Analysis of experimental epidemics of mouse-typhoid; a bacterial disease conferring incomplete immunity. Epidemiol. Infect. 39, 271-288 (1939)

24. Sweilam, N.H., Al-Mekhlafi, S.M., Albalawi, A.O., Tenreiro Machado, J.A.: Optimal control of variable-order fractional model for delay cancer treatments. Appl. Math. Model. 89(2), 1557-1574 (2021)

25. Sowndarrajan, P.T., Manimaran, J., Debbouche, A., Shangerganesh, L.: Distributed optimal control of a tumor growth treatment model with cross-diffusion effect. Eur. Phys. J. Plus 134, 463 (2019)

26. Ozdemir, N., Ucar, E.: Investigating of an immune system-cancer mathematical model with Mittag-Leffler kernel. AIMS Math. 5(2), 1519-1531 (2019)

27. De Pillis, L.G., Radunskaya, A.E., Wiseman, C.L.: A validated mathematical model of the cell-mediated immune response to the tumor growth. Cancer Res. 65, 7950-7958 (2005)

28. Mufudza, C., Walter, S., Chiyaka, E.T.: Assessing the effects of estrogen on the dynamics of chemo-virotherapy cancer. Comput. Math. Methods Med. 2012, 473572 (2012)

29. Abernathy, K., Abernathy, Z., Baxter, A., Stevens, M.: Global dynamics of a brest cancer competition model. Differ. Equ. Dyn. Syst. 3, 1-15 (2017)

30. Jarret, A.M., et al.: Experimentally-driven mathematical modeling to improve combination targeted and cytotoxic therapy for HERZ + breast cancer. Sci. Rep. 9, Article ID 12830 (2019)

31. Li, L., Liu, J.G.: A generalized definition of Caputo derivatives and its application to fractional ODEs. SIAM J. Math. Anal. 50(3), 2867-2900 (2016)

32. Abdeljawad, T., Baleanu, D.: On fractional derivatives with generalized Mittag-Leffler kernels. Adv. Differ. Equ. 2018 468 (2018)

33. Matignon, D.: Stability results for fractional differential equations with applications to control processing. Comput Eng. Syst. Appl. 2, 1-6 (1996)

34. Qian, D., Li, C., Agarwal, R.P., Wang, P.J.Y.: Stability analysis of fractional differential system with Riemann-Liouville derivatives. Math. Comput. Model. 52, 862-874 (2010)

35. Zeng, Q.S., Cao, G.Y., Zhu, X.J.: The asymptotic stability on sequential fractional order systems. J. Shanghai Jiaotong Univ. 39, 346-348 (2005) 
36. Ahmad, E., El-Sayed, A.M.A., El-Saka, H.A.A.: On some Routh-Hurwitz conditions for fractional-order differential equations and their applications in Lorenz, Rossler, Chua, and Chen systems. Phys. Lett. A 358, 1-4 (2006)

37. Courchamp, F., Berec, L., Gascoigne, J.: Allee Effects in Ecology and Conservation. Oxford University Press, Oxford (2008)

38. Bozkurt, F., Yousef, A.: Flip bifurcation and stability analysis of a fractional-order differential equation with Allee effect. J. Interdiscip. Math. 22(6), 1009-1029 (2019)

39. Allee, W.C.: Animal Aggregations: A Study in General Sociology. University of Chicago Press, Chicago (1931)

40. Lande, R.: Extinction threshold in demographic models of territorial populations. Am. Nat. 130(4), 624-635 (1987)

41. Li, X., Mou, C., Niu, W., Wang, D.: Stability analysis for discrete biological models using algebraic methods. Math. Comput. Sci. 5, 247-262 (2011)

Submit your manuscript to a SpringerOpen ${ }^{0}$ journal and benefit from:

- Convenient online submission

- Rigorous peer review

Open access: articles freely available online

- High visibility within the field

- Retaining the copyright to your article

Submit your next manuscript at $\boldsymbol{~ s p r i n g e r o p e n . c o m ~}$ 